ÖREBRO UNIVERSITET

Institutionen för naturvetenskap

\title{
ELECTRONIC STRUCTURE OF SURFACES
}

By: Ramin Lotfollahi

Supervisor: Dr. Peter Johansson

Examiner: Dr. Andreas Oberstedt

Öu-Na-Ex-NFY066-2006 


\section{Contents}

\section{Chapter 1}

Introduction 1

1.1 Nearly free electron model 1

1.2 Surface states 2

1.3 Lattice and image techniques 3

\section{Chapter 2}

Crystal structure $\quad 5$

2.1 Crystal structure $\quad 5$

2.2 Defects and dislocations 6

2.3 Crystallography 8

2.4 Surface crystallography 9

\section{Chapter 3}

One-dimensional band theory and surface states 12

3.1 The jellium model and surface electronic structure 13

3.2 One dimensional band theory 13

3.2.1 Solution of the Schrödinger equation in the bulk 14

3.2.2 Boundary conditions at the surface 16

3.2.3 Image potential 18

3.3 Numerical calculations and results 19

\section{Chapter 4}

4.1 Theoretical descriptions 23

4.2 Steps 24

4.3 Calculation method 26 
4.3.1 The density of states 26

4.3.2 The Green's functions $\quad 27$

4.3.3 Equation of motion 28

4.4 Green's function for two-dimensional terrace $\quad 30$

4.5 Results and discussions 33

Summary $\quad 35$

Appendix A: Introduction to Green's Functions 36

Appendix B: Listing of programs used for the numerical calculations

References 


\section{Preface}

The electronic structure of surfaces has become a very active area of research in the past few decades. Recent advances in experimental techniques have produced an abundant supply of reliable data on clean, well-ordered single crystal surfaces. This has urged theoretical research in the development and application of models and calculational techniques. The extent of this essay is limited to theoretical studies of the electronic structure and an simple calculation of electronic states of solid surfaces in the nearly-free and free-electron approximation. For investigation of the electronic properties of clean solid surfaces, it is helpful for the development of intuition to start with a base of detailed knowledge about simple definitions. I have therefore introduced a number of useful concepts in the first chapter. In chapter two I give a brief general view of the crystal forming and a series of technologies to identify the atomic and electronic structure of the crystals. The theory of surface states and the electronic properties at the metal surfaces have been investigated in third and fourth chapter. Finally, short discussions on the results, my working method and also some mathematical material have been placed in appendix at the end of this paper. 


\section{Chapter 1}

\section{Introduction}

Electronic properties associated with condensed matter have been studied in many years and with many different aims. The effect of technological evolutions however accelerated the theoretical and experimental developments in this area until the solid state physics and particularly electronic structure of surfaces has become one of the major research areas. As a consequence of the technological needs and the developments of new techniques in studying the crystal structure, the scientists were able to detect the electronic properties of the crystalline metal surfaces. In this context and for a better understanding of the electronic properties of solid surfaces we will investigate solid surfaces theoretically to explain a more extensive perspective of the electronic structure of surfaces.

\subsection{Nearly free electron model}

The free electron model of metals gives us better insight into the electronic properties of solid bulk of metals. In this model the allowed energy values vary continuously from zero to infinity with wave functions of the form,

$$
\psi_{k}(\vec{r})=\exp (i \vec{k} \cdot \vec{r}), \text { with } E=\frac{\hbar^{2} \vec{k}^{2}}{2 m},
$$

They are propagating waves in all directions. There is no explanation of the band structure of a crystal in this model. The band structure, however, can often be explained by the nearly free electron model that considers the effect of a weak periodic potential (Kronig-Penny model) of the ion cores. This model explains why electrons are arranged in energy bands and the behaviour of electrons in metals. The wave functions in this 
model are a product of a function $\varphi_{k}(\vec{r})$ with the same periodicity as the potential and a plane wave, $\exp (i \vec{k} \cdot \vec{r})$

$$
\psi_{k}(\vec{r})=\varphi_{k}(\vec{r}) \exp (i \vec{k} \cdot \vec{r})
$$

A wave function of this form is called Bloch function and describes electrons that propagate through the potential field of the ion cores. The wave equation can be treated in detail for a general potential, at general values of $\vec{k}$ which lies within the Brillouin zone boundary. As a consequence of interaction between the conduction electrons waves and the ion cores of the crystal, there are forbidden regions in energy space which are called energy gaps or band gaps. It means there are no electron states in these regions [Ref.2]. We will talk more detailed about this later.

\subsection{Surface states}

To discuss the electronic properties at the surface of metals we must have an understanding of the surface. In general the surface is the last few atomic layers of the solid at the nearby vacuum. In this region, the atomic geometry may depart from that of the bulk, and the effective potential seen by the electrons changes from that characteristic of the bulk to the constant vacuum level. In the direction parallel to the surface, however, we have a periodic potential similar to the bulk. In dealing with wave functions in solids, the problem is simplified by considering an infinite crystal or else a part of an infinite crystal. This is done so as to deal with wave functions which satisfy simple boundary conditions. For an idealized one-dimensional crystal in which the atomic fields are represented by square potential (The Kronig-Penny model) this simplification introduces certain features of the surface of the crystal. With this consideration Tamm found that one surface state was possible for each energy gap between the ordinary allowed bands of energies. In other words there were energy levels whose wave functions were localized at the surface of the crystal. These have been found and probed by different experimental techniques. The two most used probes of the surface spectrum are ultraviolet photoemission spectroscopy and inelastic low-energy electron scattering. Both techniques derive their surface sensitivity from the fact that the mean free path of the electrons involved in each is quite short, about 5-10 $\AA$ [Ref.1,7].

The solution of Schrödinger equation for energies in the energy gap gives an infinite number of solutions with complex $\kappa_{n}$. Waves associated with these complex wave vectors can exist just at the surface region. They are normally neglected in dealing with a bulk solid since they diverge in amplitude as $z$ goes to either $+\infty$ or $-\infty$. This means that outside the surface region, in the vacuum, the electron wave functions decay exponentially and in the bulk because of the energy gap, these waves do not exist (There is no electron states in the energy gap region). With the matching wave function model one can solve the Schrödinger equation at the surfaces, which means that the wave functions and their derivatives must match to each other. With this method and depending 
on the vacuum potential model, one or more surface electron states at the surface can be calculated. [Ref. 1,2,5,6,7].

\subsection{Lattice and image techniques}

With the discovery of X-rays, it was soon possible to image crystals, which are built of a periodic array of atoms. Studying crystal structure through the diffraction of X-rays, electron and neutron beams or other techniques shows that the crystals are made of a periodic lattice planes. The Bragg law is one of the consequences of this periodicity of the lattice.

$$
2 d \cdot \sin \theta=n \lambda
$$

Where $\theta$ is measured from the plane, $\lambda$ wavelength and $d$ spacing of parallel atomic planes [Ref.2]. A crystal is often not well-ordered and defects, dislocations and disorders in the crystal structure can affect its electronic and mechanical properties. The surface of solids exhibits interatomic distances and physical properties different from the bulk. There exist also specific properties associated to steps at a surface. Surfaces with steps have a practical interest too. It is generally easier to study the specific properties of steps on a surface that presents a regular arrangement of steps, i.e. on a vicinal surface. In general, a crystal surface cut at a small angle to a symmetry direction is called a vicinal surface. Such a miscut surface consists of terraces of the symmetry plane, separated by monatomic steps running across the sample in a preferred direction dictated by the cut.

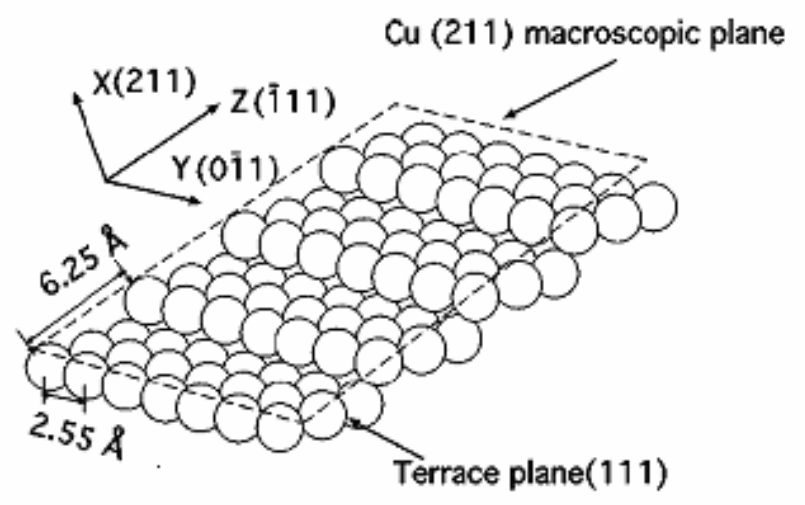

(Fig. 1.1) Structure of the $C u$ (211) vicinal surface.

The surface consists of three-atom (111) terraces separated by single-atom (100) steps.

Due to the importance of these structures, the dynamics of stepped surfaces have been the subject of several experimental investigations using modern surface-science techniques within the last several years.

Scanning tunneling microscopy is a technique developed in the eighties and allows imaging solid surfaces with extraordinary resolution. The operation of a scanning 
tunneling microscope (STM) is based on the so-called tunneling current, which starts to flow when a sharp tip approaches a conducting surface at a distance of approximately one nanometer. The tip is mounted on a piezoelectric tube, which allows tiny movements by applying a voltage at its electrodes. Thereby, the electronics of the STM system control the tip position in such a way that the tunneling current is kept constant while it is scanning a small area of the sample. This movement is recorded and can be displayed as an image of the surface topography. It should be noted, however, that STM images not only display the geometric structure of the surface, but also depend on the electronic density of states of the sample. Under ideal circumstances, the individual atoms of a surface can also be resolved and displayed [Ref.22-26].

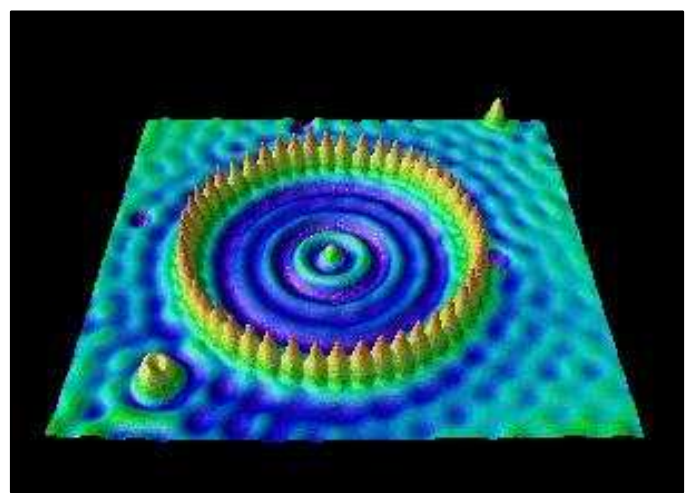

(Fig.1-1) Here is a STM image showing iron atoms adsorb on a copper (111) surface forming a so called "quantum corral" in a very low temperature (4K). Actually, the image shows the form of the local density of electron states in a box of the iron atoms. The rings in the quantum corral show the corrugations in the density of states.

Although the STM itself does not need vacuum to operate (it works in air as well as in liquids), ultrahigh vacuum is required to avoid contamination of the samples from the surrounding medium.The scanning tunneling microscope (STM) is widely used in both industrial and fundamental research to obtain atomic-scale images of metal surfaces. It provides a three-dimensional profile of the surface which is very useful for characterizing surface roughness, observing surface defects, and determining the size and conformation of molecules and aggregates on the surface.

In following chapters we will discuss more details about crystals, image techniques, the local density of states, electronic properties of surfaces and so on, but the investigation of electronic properties at the surfaces will be the main object in this essay. 


\section{Chapter 2}

\section{Crystal structure}

The beauty and symmetry of crystals have fascinated people for thousands of years. Somehow, crystals seem different from more ordinary matter. However, most solid objects are made of many tiny interlocking crystals. It is only occasionally that the individual crystals are large enough to catch our attention. As we know the crystal form develops as identical building blocks are added continuously. The building blocks here are atoms or groups of atoms. However, the atoms in a crystal form a regular repeating pattern called the crystalline lattice. In this chapter we will study the crystal structure and the techniques to image and discover crystal structure in three dimensions.

\subsection{Crystal structure}

We begin by considering a crystal and develop our knowledge about the solid crystal structure and the electronic properties. The systematic study of solid state physics began with the discovery of X-ray diffraction by crystals and the publication of a series of simple calculations of the properties of crystals and of electrons in crystals. The diffraction work proved with certainty that crystals are built up as a periodic array of atoms or groups of atoms. An ideal crystal results from the infinite repetition of identical groups of atoms. Each of these groups are called "basis", and the set of mathematical points that the basis is attached to is called "lattice".

There are 14 distinct types of lattices in three dimensions (3D) which are grouped in seven different systems, triclinic, monoclinic, orthorhombic, tetragonal, cubic, trigonal and hexagonal. There are three lattices in the cubic system: the simple cubic (sc) lattice, the body-centered cubic (bcc) lattice and the face-centered cubic (fcc) lattice (cubic closed-packed). The conventional cells of these cubic space lattices can contain one or 
more lattice points, for example a sc lattice cell contains only one lattice point, but the bcc lattice cell has two lattice points in the conventional cell and four points for a conventional cell of fcc lattice [Ref.2]. Fig.3-1 shows the conventional cells of these cubic lattices.

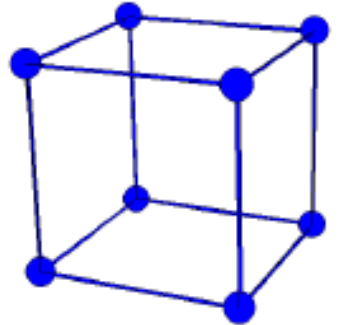

simple cubic (sc)

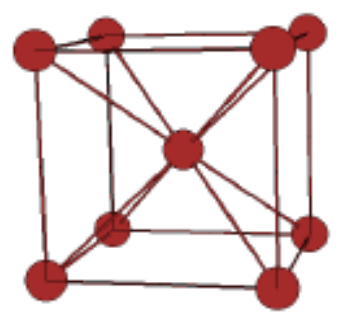

body-centered cubic (bcc)

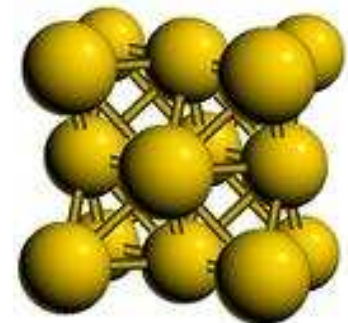

face-centered cubic (fcc, $c c p)$

(Fig.2-1)

\subsection{Defects and dislocations}

It is useful to think about solids in terms of a regular repeating pattern of planes of particles. But it is important to recognize that solids are seldom perfectly ordered, to be exact; defects and dislocations are some of the usual causes of disorder in crystals. Some basic mechanisms for introducing a point defect into the structure of a solid are:

- Vacancies are sites that are usually occupied by an atom but now are unoccupied. If a neighbouring atom moves to occupy the vacant site, the vacancy moves in the opposite direction, to the site which used to be occupied by the moving atom. The stability of the surrounding crystal structure guarantees that the neighbouring atoms will not simply collapse around the vacancy. (In some materials, neighbouring atoms actually move away from a vacancy, because they can better form bonds with atoms in the other directions.)

- Interstitials are atoms which occupy a site in the crystal structure at which there is usually not an atom. They are generally high-energy configurations. Small atoms can in some crystals occupy interstices without high energy penalty, such as hydrogen in palladium.

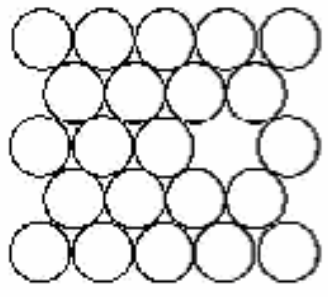

(a)

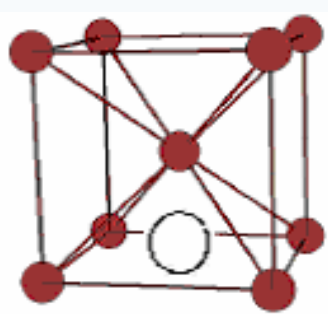

(b)

(Fig.2-2) schematically shows (a) a vacancy and (b) an interstitial defect 
- Impurities occur because a material is never $100 \%$ pure. In the case of an impurity, the atom is often incorporated at a regular atomic site in the crystal structure. This is neither a vacant site nor is the atom on an interstitial site and it is called a substitution defect. The atom is not supposed to be anywhere in the crystal, and is thus an impurity.

- Anti-site defects occur in an ordered alloy. For example, some alloys have a regular structure in which every other atom is a different species. For example assume that type $\mathrm{A}$ atoms sit on the cube corners of a cubic lattice, and type B atoms sit in the centre of the cubes. If one cube has an $\mathrm{A}$ atom at its centre, the atom is on a site usually occupied by an atom, but it is not the correct type. This is neither a vacancy nor an interstitial, nor an impurity, but anti-site defect.

- Complexes can form between different kinds of point defects. For example, if a vacancy encounters an impurity, the two may bind together if the impurity is too large for the lattice. Interstitials can form 'split interstitial' or 'dumbbell' structures where two atoms effectively share an atomic site, resulting in neither atom occupying the actual site.

- Dislocations are line defects around which some of the atoms of the crystal lattice are misaligned. There are two basic types of dislocations, the EDGE dislocation and the SCREW dislocation. ("MIXED" dislocations combining aspects of both types are also common).

Edge dislocations are caused by the termination of a plane of atoms in the middle of a crystal. In such a case, Fig.3-2, the adjacent planes are not straight, but instead bend around the edge of the terminating plane so that the crystal structure is perfectly ordered on either side. The screw dislocation is more difficult to visualise, but basically comprises a structure in which a helical path is traced around the linear defect (dislocation line) by the atomic planes of atoms in the crystal lattice. It is similar to a spiral stair case.

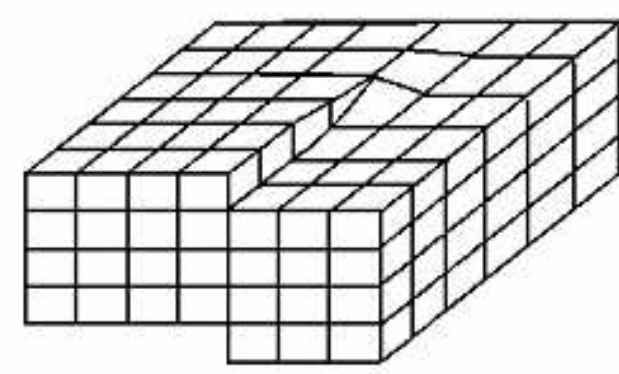

(a)

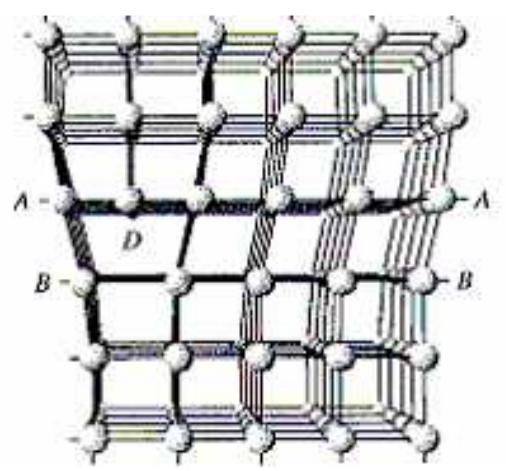

(b)

(Fig2-3) schematic shows structure of a screw dislocation in (a) and an edge dislocation in (b).

Dislocations can move if the atoms from one of the surrounding planes break their bonds and rebinds with the atoms at the terminating edge. It is the presence of dislocations and their ability to readily move (and interact) under the influence of stresses 
induced by external loads that leads to the characteristic malleability of metallic materials. Dislocations can be observed using transmission electron microscopy, field ion microscopy and atom probe techniques. Disinclinations are line defects corresponding to "adding" or "subtracting" an angle around a line. Basically, this means that if you track the crystal orientation around the line defect, you get a rotation [Ref.2].

\subsection{Crystallography}

In the last decade the ability of materials scientists to synthesize materials has enabled the development of new technologies with applications that range from the spectacular to the ordinary. For example, X-ray mirrors composed of alternating, thin (less than 20-nanometer) films of molybdenum and silicon constitute the optics that are used to produce high-resolution pictures of the sun. Optoelectronic components composed of alternating atomic layers of different elements are the devices that enable us to extract information from video compact disks and to generate and detect transoceanic telephone signals by fiberoptic cables. The alternating, ultra thin layers of cobalt and iron in new high-density magnetic storage heads, and increasingly miniature microelectronics, are fundamental constituents of powerful desktop computers, portable laptops, and pocket-size wireless telephones. The smaller these devices become, the more their performance depends on the atomic ordering of their component materials. Such details include the arrangement of atoms in crystal structures and the presence, size, and density of grain boundaries, impurities, dislocations, or other imperfections.

To identify the effect of atomic arrangement on material performance, materials scientists use a variety of techniques. X-ray diffraction, transmission electron microscopy and scanning tunnelling microscopy are some of these techniques. Diffraction occurs as waves interact with a regular structure whose periodicity is about the same as the wavelength. The phenomenon is common in physics, and occurs on all length scales. For example, light can be diffracted by a grating having scribed lines spaced on the order of a few thousand angstroms, about the wavelength of light. X-rays happen to have wavelengths on the order of a few angstroms, the same as typical interatomic distances in crystalline solids. That means X-rays can be diffracted from minerals which, by definition, are crystalline and have regularly repeating atomic structures. When certain geometric requirements are met, X-rays scattered from a crystalline solid can constructively interfere, producing a diffracted beam.

The Transmission Electron Microscope (TEM) allows the user to determine the internal structure of materials, either of biological or non-biological origin. The energy of the electrons in the TEM determines the relative degree of penetration of electrons in a specific sample, or alternatively, influence the thickness of material from which useful information may be obtained. Because of the high spatial resolution obtained, TEMs are often employed to determine the detailed crystallography of fine-grained, or rare, materials. Thus, for the physical and biological sciences, TEM is a complementary tool to conventional crystallographic methods such as X-ray diffraction. 
The scanning tunnelling microscope (STM) provides a picture of the atomic arrangement of a surface by sensing corrugations in the electron density of the surface that arise from the positions of surface atoms. A finely sharpened tip usually made of tungsten wire is first positioned within 2 nanometers of the specimen by a piezoelectric transducer, a ceramic positioning device that expands or contracts in response to a change in applied voltage. This arrangement allows us to control the motion of the tip with subnanometer precision. At this small separation, as explained by the principles of quantum mechanics, electrons "tunnel" through the gap, the region of vacuum between the tip and the sample. If a small voltage (bias) is applied between the tip and the sample, a net current of electrons (the "tunnelling current") flows through the vacuum gap in the direction of the bias. For a suitably sharpened tip (one that terminates ideally in a single atom) the tunnelling current is confined to a channel with a width of a few Angstroms. The remarkable spatial resolution of the STM is due to this lateral confinement of the current [Ref.22-25].

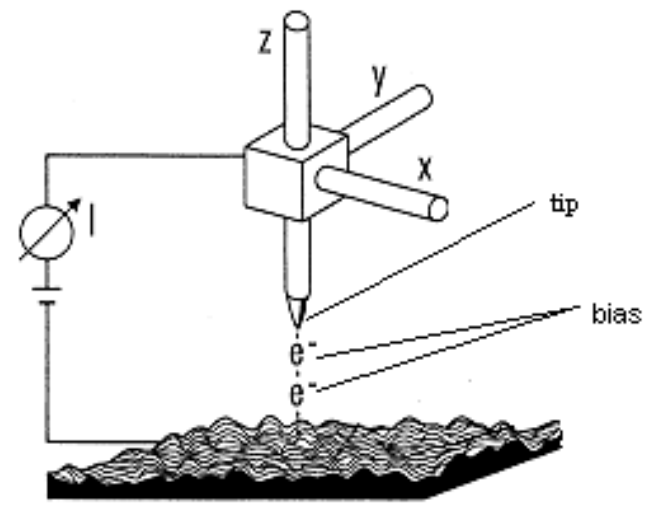

(Fig.2-4) an image of the STM technique. The probe tip as held by tripod, which consists of three piezoelectric cylinders that expand or contract in the directions $(x, y, z)$ shown to displace the tip.

In this context, the STM offers a new opportunity for direct diagnosis of how the processing conditions affect the atomic details of surfaces. This combination of surface diagnostics is used to study the structural development of metal surfaces and electron density for different appearances which is the central topic in this essay.

\subsection{Surface crystallography}

The surface of a crystalline solid in vacuum is generally defined as the few outermost atomic layers of the solid that differ significantly from the bulk. The surface may be entirely clean or it may have foreign atoms deposited on it or incorporated in it. However, in this essay we consider that the surface of solid, particularly metals, is clean. For a better understanding of a solid surface we need knowledge about atoms and their place (which atoms and where). Actually it is the geometrical arrangement of the surface atoms that determines the electronic and magnetic properties at the surface. The bulk structure problem is resolved by $\mathrm{x}$-ray diffraction but unfortunately the extremely large penetration depth limits their routine use for surface crystallography. A number of techniques have been developed for surface-specific structural examination. Some of these common techniques will be discussed below. 
The surface structure is in general periodic in two dimensions (but needless to say there is no periodicity in the third direction). At a surface, the electrons are free to rearrange their distribution in space to lower their energy. The net force on the ions points primarily into the crystal and a contraction relaxation of the surface plane occurs until equilibrium is re-established. In this point an STM image can thus give a complete picture of the local density of states. The local density of states (LDOS) represents the amount of electrons that exist at specific values of energy. Keeping the gap distance constant, measuring the current change with respect to the bias voltage can probe the LDOS of the sample. Moreover, changing the polarity of bias voltage can get local occupied and unoccupied states. In Fig.2-5, when the tip is negatively biased, electrons tunnel from the occupied states of the tip to the unoccupied states of the sample. If the tip is positively biased, electrons tunnel from the occupied states of sample to the unoccupied states of the tip.

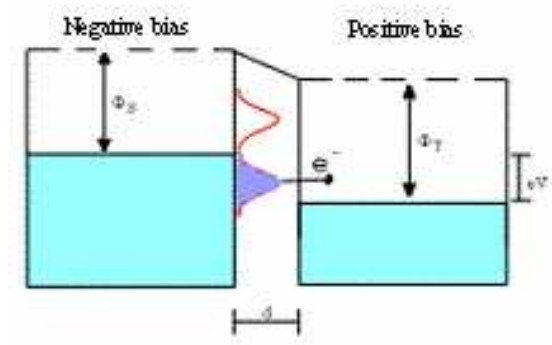

(a)

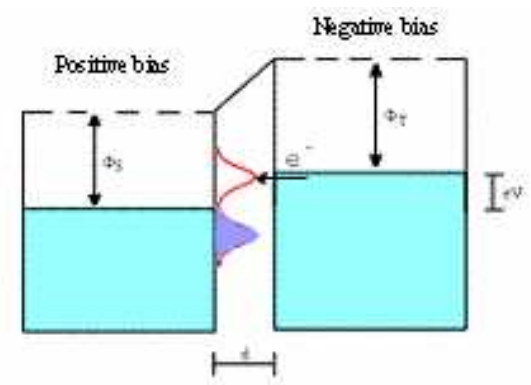

(b)

(Fig.2-5) a demonstration of STM technique (a) with positive biased tip that the electrons tunnel from the sample to the unoccupied states of the tip and $(b)$ negative biased tip with tunnelled electrons from the tip to the unoccupied states of the sample

Surfaces of planes nominally of high indices may be built up of low index planes separated by steps one or two atoms in height (Fig.1-1). Such terrace-step arrangements are important in evaporation and desorption because the attachment energy of atoms is often low at the steps and at kinks in the steps. The chemical activity of such sites may be high. Double and triple beams of diffraction in LEED experiments may detect the presence of periodic arrays of steps. Long experience with diffraction methods in the bulk suggests a search for a similar methodology at the surface. As always, a diffraction experiment designed for crystal structure analysis requires a probe with de Broglie wavelength less than typical interatomic spacing, for example about $1 \AA$, which gives a kinetic energy about $E=(h / \lambda)^{2} / 2 m \cong 150 e V$. Electrons with energies in the range 20-500 $\mathrm{eV}$ that is elastically backscattered from the crystal surface will form a diffraction pattern that is the Fourier transform of the surface atom arrangement. This forms the basis for "low energy electron diffraction" (LEED) from solid surfaces. 


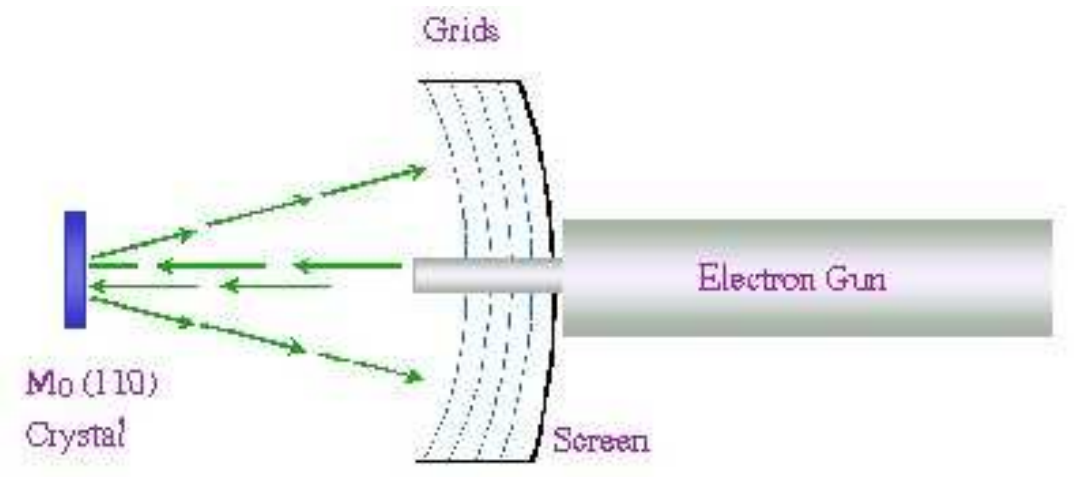

(Fig.2-6)An image of LEED techniques

An image of the low energy electron diffraction, LEED in Fig.3-5 shows schematically how this technique works. Electrons from an electron gun diffract off the sample and head back towards the electron gun and the grids surrounding it. The two middle grids are set at energy slightly less than the electron beam to prevent any inelastically scattered electrons from reaching the screen. The screen is biased positive between 0 and $7 \mathrm{keV}$ accelerating the electrons towards it. The screen is coated in phosphor, which glows green when electrons hit it. The resulting LEED pattern is viewed from the rear end of the apparatus. 


\section{Chapter 3}

\section{One-dimensional band theory and surface states}

The theory of surface states has been studied for many years. Tamm was the first to consider the wave function for a semi-infinite crystal with a simplified piecewise constant periodic potential (Kronig-Penney). He found that it was possible to have energy levels whose wave functions were localized at the surface of the crystal and one surface level was possible for each energy gap between the ordinary allowed bands of energies. Shockley followed Tamm's consideration and found that there are more electronic states localized at the surface region as a consequence of the vacuum potential model [Ref.5]. Both of them used the method of matching the wave function and its derivative at crystal surface.

We will make considerable use of simple methods to exhibit the most characteristic features of surface electronic structure. We know that there are different interactions between atoms (ions and electrons). The Hamiltonian that describes the surface electronic structure contains three terms; the kinetic energy of electrons, the ion-electron attraction and the electron-electron repulsion, ignoring ion motion.

$$
H=\text { Kinetic energy }+ \text { ion-electron attraction + electron-electron repulsion }
$$

Here we are going to solve this problem within a simple one-dimensional nearly-free electron model which neglects the electron-electron interaction. The effective potential includes only the ion cores and an image surface barrier [Ref.1]. 


\subsection{The jellium model and surface electronic structure}

In this chapter I will give a basic solution of Schrödinger equation in one dimension. While we must assume that we have at least a passing familiarity with common concepts and calculation techniques in the bulk solids, a few general concepts related to surface electronic structure will be introduced below.

A basic concept that we already mentioned is the surface region, which refers to the volume of space containing the last few atomic layers of the solid. In this region the atomic geometry and the effective potential may not be the same as in the bulk. If we know the position of all atoms (almost) we get the Hamiltonian that describes the surface electronic structure as a function of the kinetic energy, ion-electron attraction and electron- electron repulsion. For present purpose, we rephrase the exact solution to the electronic structure problem in term of the "self-consistent "calculation. Self-consistency involves redistribution of charge throughout the cluster until a minimum energy is reached, thereby hopefully producing an exact reproduction of the charge distribution in an equivalent real system. In this model, which can be used for the case of simple metals, we use an approximation that replaces the ions by a uniform semi-infinite positive charge background of density. This method is called the jellium model and was used for the first time by Bennett, Duke and Smith [Ref.1\&6].

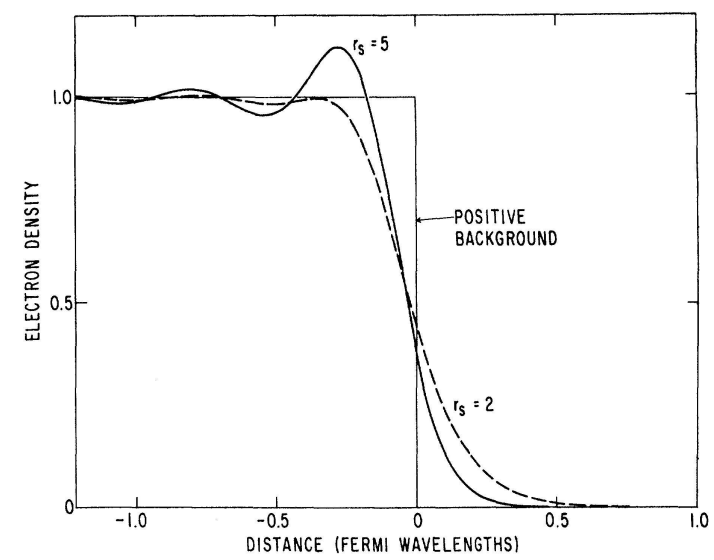

The positive background charge density, $\tilde{\mathrm{n}}$, often is expressed in term of an inverse sphere volume $(4 \cdot \pi / 3) \cdot r_{s}^{3}=1 / \tilde{n}$. Typical values of $r_{s}$ range from about two to five. (N.D.Lang \& W.Kohn)

(Fig.3-1) self-consistent charge density near metal surface for $r_{s}=2$ and $r_{s}=5$

(Uniform positive background model)

The jellium description of a metal surface is a one- dimensional model that neglects the details of the electron-ion interaction and emphasizes the nature of the smooth surface barrier.

\subsection{One dimensional band theory}

The one-dimensional band structure approach to surface electronic structure emphasizes the lattice aspects of the problem and simplifies the form of the surface barrier. The basic 
argument of the band structure models is the influence of a boundary condition for the Schrödinger equation that reflects the presence of a free surface. These boundary conditions lead to the existence of surface states for the free-electron model.

\subsubsection{Solution of the Schrödinger equation in the bulk}

Now it is time to find a basic solution of Schrödinger equation that leads to an understanding of band structure in solids. The one-dimensional nearly free-electron model gives:

$$
\left[-\frac{d^{2}}{d z^{2}}+V(z)\right] \psi(z)=E \psi(z),
$$

Here $V(z)$ is a weak periodic pseudopotential,

$$
V(z)=-V_{0}+2 V_{g} \cos g z
$$

and $g=2 \pi / a$ is the shortest reciprocal lattice vector of the chain. We know the solution of these problems especially in the bulk which we have already seen. In the free electron model $\left(V_{g}=0\right)$ the allowed energy values are distributed continuously from zero to infinity and in the case of nearly-free electron model, as we have seen (C.Kittel) the band structure of a crystal can be explained because the band electrons are treated as perturbed only weakly by the periodic potential of the ion cores.

From before we know that the wave function of an electron in a periodic potential may be expressed as a Fourier series summed over all values of the wave vectors permitted by boundary conditions. For our purpose, however, we consider a superposition of two plane waves, which is the most important part of the Fourier series at the first Brillouin zone boundary [Ref.1\&2]:

$$
\psi(z)=\alpha \cdot e^{i k z}+\beta \cdot e^{i \cdot(k-g) z},
$$

Substituting this function into our Schrödinger equation leads to;

$\left[-d^{2} / d z^{2}-V_{0}+2 V_{g} \cos g z\right] \cdot\left(\alpha \cdot e^{i k z}+\beta \cdot e^{i(k-g) z}\right)=E \cdot\left(\alpha \cdot e^{i k z}+\beta \cdot e^{i(k-g) z}\right)$,

If we use, $\cos g z=\frac{e^{i g z}+e^{-i g z}}{2}$ and shift the zero of the energy scale so that it coincides with $-V_{0}$, then we have;

$$
\left.\left[\left(k^{2}-E\right) \cdot \alpha+\beta \cdot V_{g}\right] e^{i k z}+\left\{(k-g)^{2}-E\right] \cdot \beta+\alpha \cdot V_{g}\right\} e^{i(k-g) z}
$$

$$
+\alpha \cdot V_{g} e^{i(k+g) z}+\beta \cdot V_{g} e^{i(k-2 g) z}=0,
$$


The two last terms in (3-6), are placed in the second Brillouin zone and are insignificant for this solution. Therefore we can write our equation in its secular shape;

$\left[\begin{array}{cc}\vec{k}^{2}-E & V_{g} \\ V_{g} & (\vec{k}-g)^{2}-E\end{array}\right]\left[\begin{array}{l}\alpha \\ \beta\end{array}\right]=\left[\begin{array}{l}0 \\ 0\end{array}\right] \quad$ (Central equation)

This equation has none-trivial solutions only when the determinant of the matrix is zero. If the wave vector $\vec{k}$ should be written in terms of its deviation from the Brillouin zone boundary, $\vec{k}=g / 2+\kappa$ then we have the solution for the energy eigenvalues;

$\left(\vec{k}^{2}-E\right) \cdot\left[(\vec{k}-g)^{2}-E\right]-V_{g}^{2}=0 \Rightarrow$

$\left[(g / 2+\kappa)^{2}-E\right] \cdot\left\{[(g / 2+\kappa)-g]^{2}-E\right\}-V_{g}=0 \Rightarrow$

$E=(g / 2)^{2}+\kappa^{2} \pm\left(g^{2} \cdot \kappa^{2}+V_{g}^{2}\right)^{1 / 2}$,

And for the wave vectors,

$\kappa^{2}=\left[(g / 2)^{2}+E\right] \pm \sqrt{g^{2} \cdot E+V_{g}^{2}}$,

For the one-dimensional semi-infinite nearly-free electron model $E\left(\kappa^{2}\right)$ shows the familiar energy gap at the Brillouin zone boundary. However, $E\left(\kappa^{2}\right)$, is a continuous function of $\kappa^{2}$ if one allows $\kappa$ to take imaginary values.

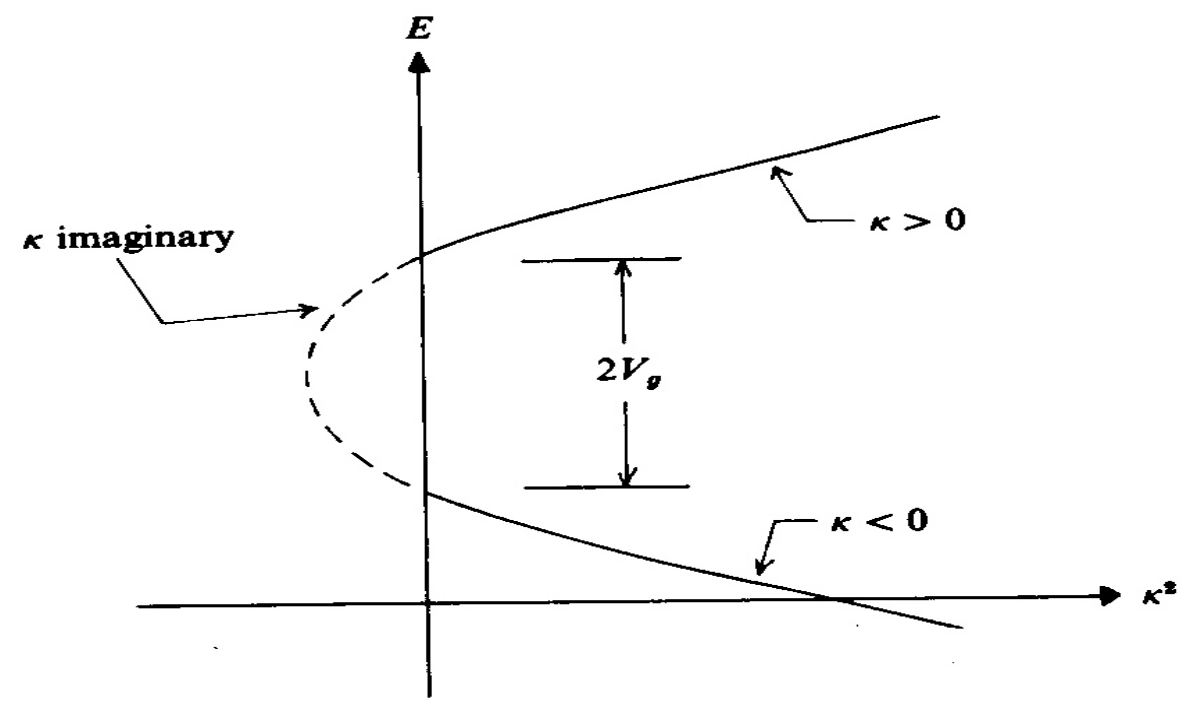

(FIG.3-2) $E\left(\kappa^{2}\right)$ for the one-dimensional semi-infinite nearly free electron model [Ref.1] 
Now when we have the energy eigenvalues, it is obvious that the wave function would be found by solving the "Central equation":

$\left(\vec{k}^{2}-E\right)^{2} \cdot \alpha+V_{g} \cdot \beta=0$

$V_{g} \cdot \alpha+\left[(\vec{k}-g)^{2}-E\right] \cdot \beta=0$,

In these equations $\alpha, \beta$ must be chosen so that the both (3-9a) and (3-9b) are valid. The result is simplified if we represent $\alpha=A^{\prime} \cdot e^{-i \delta}$ and $\beta=A^{\prime} \cdot e^{i \delta}$, there $A^{\prime}$ is a constant and $\delta$ is "phase shift" between the forward-moving waves and backward-moving waves. Actually $\delta$ varies smoothly in energy band gap between $\pi / 2$ and zero.

$\beta / \alpha=\frac{E-\vec{k}^{2}}{V_{g}} \Rightarrow \exp (2 i \delta)=\frac{E-\vec{k}^{2}}{V_{g}}$,

$\psi_{\kappa}(z)=A^{\prime} \cdot e^{-i \delta} \cdot \exp [i \cdot(g / 2+\kappa) \cdot z]+A^{\prime} \cdot e^{i \delta} \cdot \exp [i \cdot(\kappa-g / 2) \cdot z]$, with $A=A^{\prime} / 2$ we have;

$\psi_{\kappa}(z)=A \cdot e^{i \kappa z} \cdot \cos \left(\frac{1}{2} \cdot g \cdot z-\delta\right)$

This wave function is a product of an exponentially decaying function for imaginary $\kappa$ and a periodic function and increases for positive $\mathrm{z}$ and decreases for negative values of $z$. This is acceptable since it will be matched (at $z=a / 2$ ) onto a function that describes decay of the wave function in the vacuum. In this way we get a wave function describing a surface state.

\subsubsection{Boundary conditions at the surface}

We have already seen that the wave function for the bulk increases when $z$ goes to positive values, but it must also match the vacuum function, which is a decreasing exponential wave function. This means that these two functions and their derivatives must be continuous at the surface. For the last layer of the crystal, this point counts to be at the half of the crystal lattice constant $a$;

$$
\begin{array}{ll}
\psi(z)=A e^{i \cdot k \cdot z} \cos \left(\frac{1}{2} \cdot g \cdot z-\delta\right) & z\left\langle\frac{a}{2}\right. \\
\psi(z)=C e^{-q \cdot z} & z>\frac{a}{2} \quad, \text { where } q^{2}=V_{0}-E,
\end{array}
$$


If the logarithmic derivative of $\psi_{\kappa}(z)$ can be made continuous at $z=a / 2$, an electronic state exists that is localized near the surface of the lattice chain. The energy of this surface state lies in the bulk energy gap.

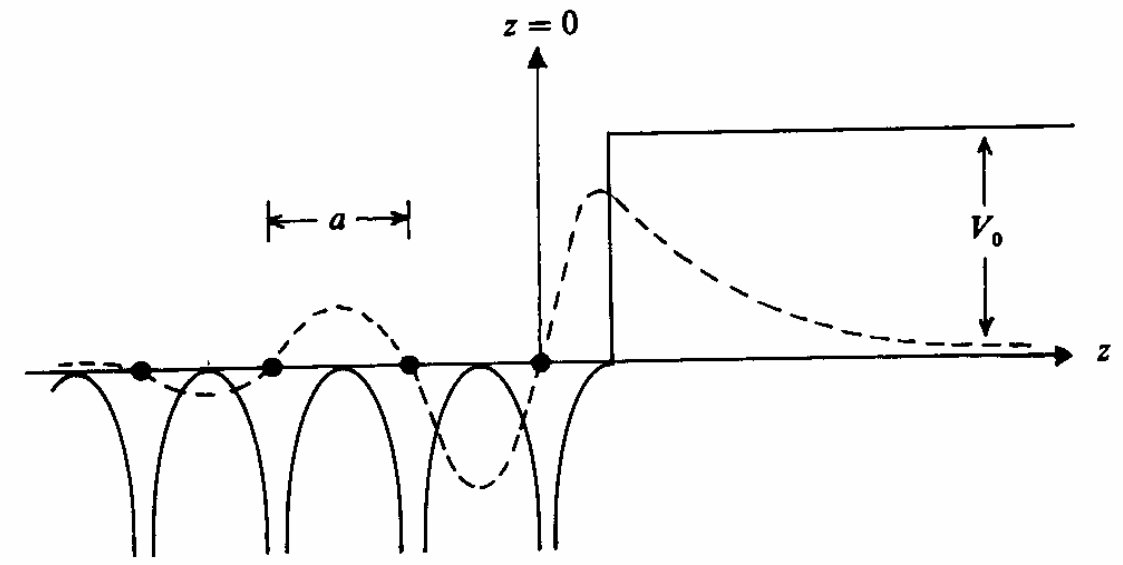

(Fig.3-3) One-dimensional semi-infinite lattice model potential and an associated surface state

$$
\begin{aligned}
& \frac{d \psi^{\text {in }} / d z}{\psi^{\text {in }}}(a / 2)=\frac{d \psi^{\text {out }} / d z}{\psi^{\text {out }}}(a / 2) \Rightarrow \quad \frac{\left(\psi^{\text {in }}\right)^{\prime}}{\psi^{\text {in }}}(a / 2)=\frac{\left(\psi^{\text {out }}\right)^{\prime}}{\psi^{\text {out }}}(a / 2) \Rightarrow \\
& {\left[\begin{array}{c}
e^{\frac{i \cdot \kappa \cdot a}{2}} \cdot \cos \left(\frac{1}{4} \cdot g \cdot a-\delta\right) \\
i \cdot \kappa \cdot e^{\frac{i \cdot \kappa \cdot a}{2}} \cdot \cos \left(\frac{1}{4} \cdot g \cdot a-\delta\right)-\frac{g}{2} \cdot e^{\frac{-q \cdot a}{2}} \cdot \sin \left(\frac{1}{4} \cdot g \cdot a-\delta\right) \\
q \cdot e^{\frac{-q \cdot a}{2}}
\end{array}\right] \cdot\left[\begin{array}{l}
A \\
C
\end{array}\right]=\left[\begin{array}{l}
0 \\
0
\end{array}\right] \Rightarrow}
\end{aligned}
$$

$\left[\begin{array}{cc}D 1 & D 2 \\ D 3 & D 4\end{array}\right] \cdot\left[\begin{array}{l}A \\ C\end{array}\right]=\left[\begin{array}{l}0 \\ 0\end{array}\right]$

This secular equation has solution if and only if, $\operatorname{det}\left[\begin{array}{cc}D 1 & D 2 \\ D 3 & D 4\end{array}\right]=0 \Rightarrow$ $D 1 \cdot D 4-D 2 \cdot D 3=0 \quad \Rightarrow$ $e^{-\frac{q \cdot a}{2}} \cdot e^{\frac{i \cdot \kappa \cdot a}{2}} \cdot\left[q \cdot \cos \left(\frac{1}{4} \cdot g \cdot a-\delta\right)+i \cdot \kappa \cdot e^{\frac{i \cdot \kappa \cdot a}{2}} \cdot \cos \left(\frac{1}{4} \cdot g \cdot a-\delta\right)-\frac{g}{2} \cdot \sin \left(\frac{1}{4} \cdot g \cdot a-\delta\right)\right]=0$

This equation determines $E$. Note that $\kappa, q$ and $\delta$ are functions of the energy. For each $\kappa$ and energy $E$, the electron states will be given by the solution of Schrödinger equation for the bulk potential (periodic in direction normal to the surface). The electron states exist if the wave functions $\psi_{\kappa}(z)$ and their derivatives are continuous at the surface. 
We should not forget that there are an infinite numbers of solutions for complex $\kappa$ but just those that satisfy the continuous boundary conditions at the surface are important for our case.

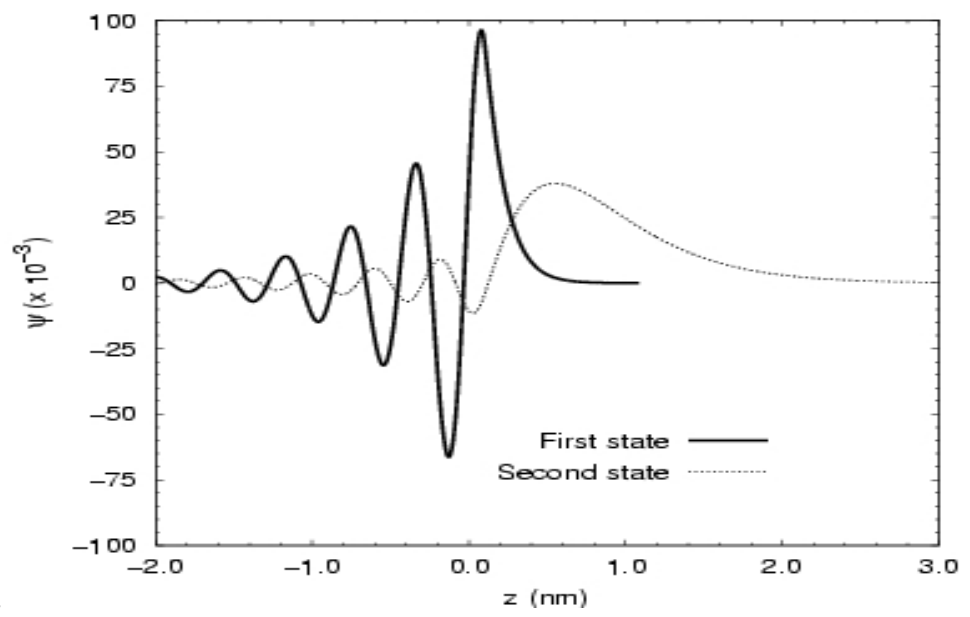

(Fig.3-4) the wave function associated to surface, $z=0$ corresponds to solid atomic layer position, $a=2.08 \AA$ for $\mathrm{Cu}(111)$

\subsubsection{Image potential}

If an electron has enough energy to exist in the neighbourhood of a metal surface, the presence of the electron affects the charge distribution on the metal surface. A positive charge will be distributed along the metal surface with the same magnitude. To calculate the electrostatic interaction force produced by the electron and the positive charge distribution we use one positive charge with the same magnitude as the positive charge distribution and with the same distance from the surface, and calculate the electrostatic force between these two point charges. It means that the electron generates a hole in the crystal at the same distance from the surface in opposite direction. This positive point charge is called the image charge. If the electron is at a distance $z$ from the surface, the distance between the electron and its image will be $2 z$. At this case the electrostatic force of attraction between the two charge particles $e^{+}$and $e^{-}$will be;

$$
F=\frac{1}{4 \pi \varepsilon_{0}} \frac{e^{-} \cdot e^{+}}{d^{2}},
$$

Here $d=2 z$ is the distance between the electron and its image. Then,

$$
F=\frac{1}{4 \pi \varepsilon_{0}} \frac{e^{2}}{(2 z)^{2}} \quad \Rightarrow \quad F=\frac{1}{16 \pi \varepsilon_{0}} \frac{e^{2}}{z^{2}},
$$

The image potential energy is therefore the integral of the electrostatic force from infinity to the distance $z$ from the surface, i.e.

$$
\begin{aligned}
& V(z)=\int_{z}^{\infty} F(z) d z \quad \Rightarrow \quad V(z)=\frac{e^{2}}{16 \pi \varepsilon_{0}} \int_{z}^{\infty} \frac{1}{z^{2}} d z \quad \Rightarrow \\
& V(z)=-\frac{1}{16 \pi \varepsilon_{0}} \frac{e^{2}}{z},
\end{aligned}
$$




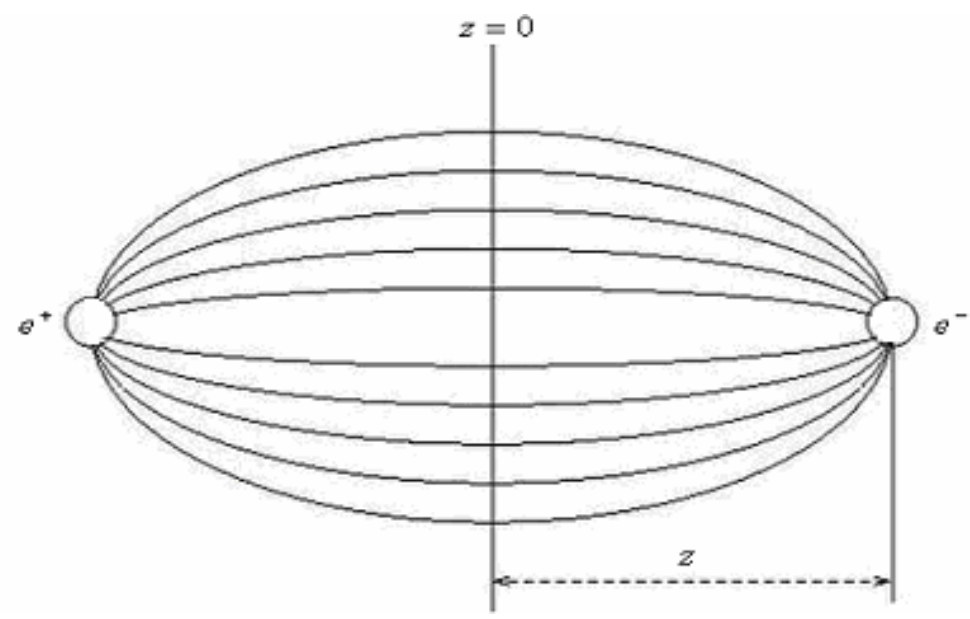

(Fig3-5) Field lines for an electron and its image in the crystal

Then the electrons outside the surface would be affected by a constant vacuum potential plus the image potential (3-17). This is a potential that changes with the distance from the surface and for large distances approaches to a constant vacuum level (zero).

\subsection{Numerical calculations and results}

To solve the Schrödinger equation (3-2) one needs to know the potential, $V(z)=-V_{0}+2 V_{g} \cos g z$ because a detailed calculation of this equation would require the knowledge of the bulk structure. In this purpose I used copper $(\mathrm{Cu}(111))$ as an example. $\mathrm{Cu}(111)$ is one of the most studied metal surfaces. This surface represents a simple kind of potential and on this surface the energy gap is located below the vacuum level and the first state (actually there is just one state if the vacuum potential barrier is high enough) is at about the middle of this gap. $V_{0}=11,562 \mathrm{eV}, V_{g}=2,5 \mathrm{eV}$ and $a=2.08 \mathrm{~nm}$ are relevant parameter values for the copper (111).

In the vacuum, however, the potential is constant and the wave function is an exponentially decaying function. All details about this wave function can be calculate by (3-11b) and (3-12) i.e. matching the wave functions and their derivatives at the surface $(z=a / 2)$.

\begin{tabular}{|c|c|c|c|}
\hline level & Energy $(\mathrm{eV})$ & Potential $(\mathrm{eV})$ & Gap $(\mathrm{eV})$ \\
\hline 1 & 8,003 & $V_{0}=11,562 \mathrm{eV}$ & $E_{\text {midgap }}=8,797 \mathrm{eV}$ \\
\hline & & $2 V_{g}=5 \mathrm{eV}$ & $E_{g b}=6,300 \mathrm{eV}$ \\
\hline & & & $E_{g t}=11,293 \mathrm{eV}$ \\
\hline
\end{tabular}

Tab.3-1 the only Tamm state for the square potential model. $E_{g b}$ and $E_{g t}$ are the lowerand upper edge of the gap and $E_{\text {midgap }}$ is the energy of its middel 


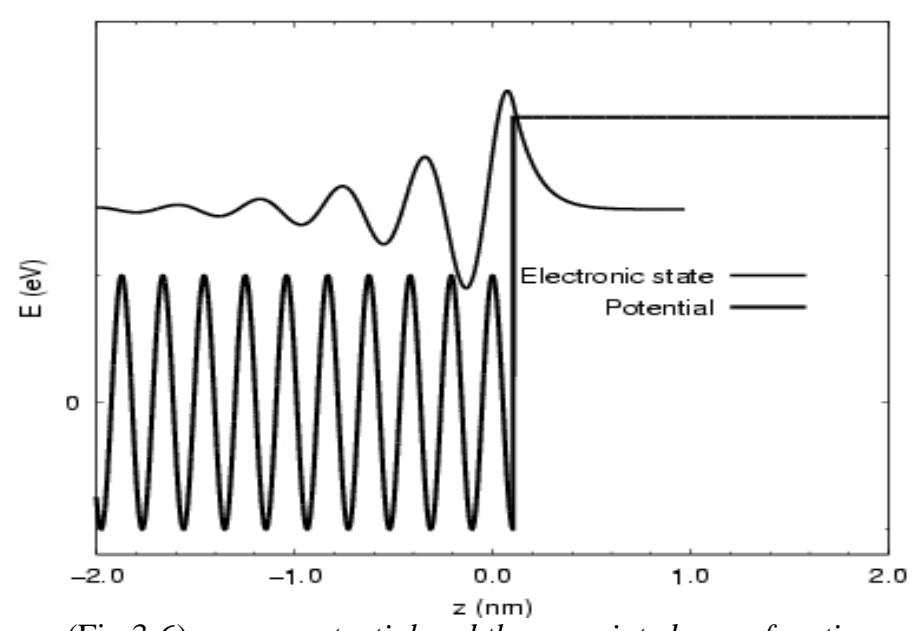

(Fig.3-6) square potential and the associated wave function

Fig 3-6 shows the wave function associated with the square potential model. This model potential was considered by Tamm and therefore these surface states are called "Tamm states". The charge density localization shows that these states are localized mainly at the surface atomic layer. For a square model potential, irrespective of vacuum level, I always got just one surface state which agrees with the Tamm state. In this case there is one electron state whose energy lies at about the middle of the band gap. This is shown in Tab.3-1. The higher the vacuum level, the closer the energy level is to the middle of the band gap.

With a image potential that is the result an electron located in the neighbourhood of a surface, the wave function changes significantly compared to our first case. In this case the potential varies with distance and therefore to calculate the wave function we use numerical solution [Ref.21]. This numerical solution gives us the shape of the wave function in the vacuum and as I previously mentioned these two wave functions (twoplane wave function and function in the vacuum) must be continuous at $z=a / 2$ (i.e. logarithmic derivative). Tab.3-2 shows the values of the image states for the vacuum model potential.

\begin{tabular}{|c|c|c|c|}
\hline level & Energy $(\mathrm{eV})$ & Potential & Gap \\
\hline 1 & 7,407 & $V_{0}=11,562 \mathrm{eV}$ & $E_{\text {midgap }}=8,797 \mathrm{eV}$ \\
\hline 2 & 11,161 & $2 V_{g}=5 \mathrm{eV}$ & $E_{g b}=6,300 \mathrm{eV}$ \\
\hline & & & $E_{g t}=11,293 \mathrm{eV}$ \\
\hline
\end{tabular}

Tab. 3-2 the Tamm state (level 1) and the image state (level 2) of the image potential model 


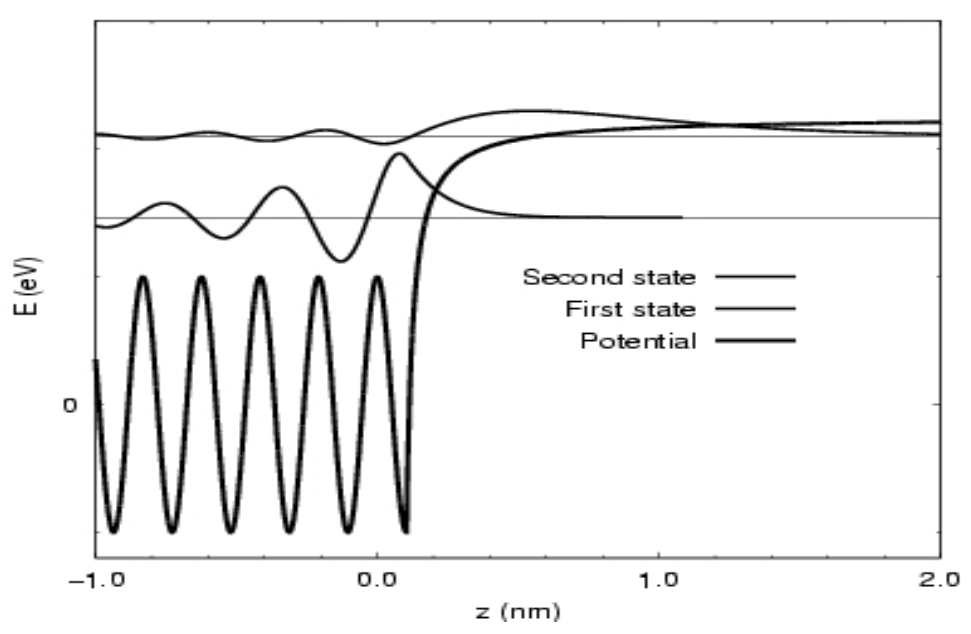

(Fig.3-7) image potential and the associated wave functions

Fig.3-7 and 3-8 shows the wave functions and the probability amplitude of the $n=1$ surface state and $n=2$ image state obtained with the use of the image potential. In these figures we see a corresponding potential energy diagram for an electron in front of a $\mathrm{Cu}$ (111) surface. For this surface the energy gap lies below the vacuum level and the surface state is located almost in the middle of the band gap but the image state (the only image state) is at the top of this gap just below the upper edge of the gap. The maximum of the probability density for the image potential state shown in Fig.3-8 is several Angstrom away from the surface. If the vacuum level is high and the energy gap lies below the vacuum level, we have one electron state that is similar the to the one of the square potential model but this state has lower energy, 7,523 (eV) compare to the surface state of the squre potential model, 8,003 (eV) (Tab.3-3).

\begin{tabular}{|c|c|c|c|}
\hline level & Energy $(\mathrm{eV})$ & Potential $(\mathrm{eV})$ & Gap $(\mathrm{eV})$ \\
\hline 1 & 7,523 & $V_{0}=12,00 \mathrm{eV}$ & $E_{\text {midgap }}=8,797 \mathrm{eV}$ \\
\hline & & $2 V_{g}=5 \mathrm{eV}$ & $E_{g b}=6,300 \mathrm{eV}$ \\
\hline & & & $E_{g t}=11,293 \mathrm{eV}$ \\
\hline
\end{tabular}

Tab.3-3 The Tamm state for high image potential case

For the case that the upper edge of the energy gap lies above the vacuum level, there are many electron states with energies just below the vacuum level. In this case, which is shown in Tab.3-4, the image states are localized far outside the surface in the vacuum.

A surface state near the centre of the gap is highly localized in the surface region, these states as we already seen are Tamm states. However one with its energy near the edge of the gap will have a lot of its weight in a slowly decaying tail in the vacuum. Thus a state merging into the edge of the gap might gradually fade away in terms of its amplitude at the surface, rather than unexpectedly vanish [Ref.7-9]. 


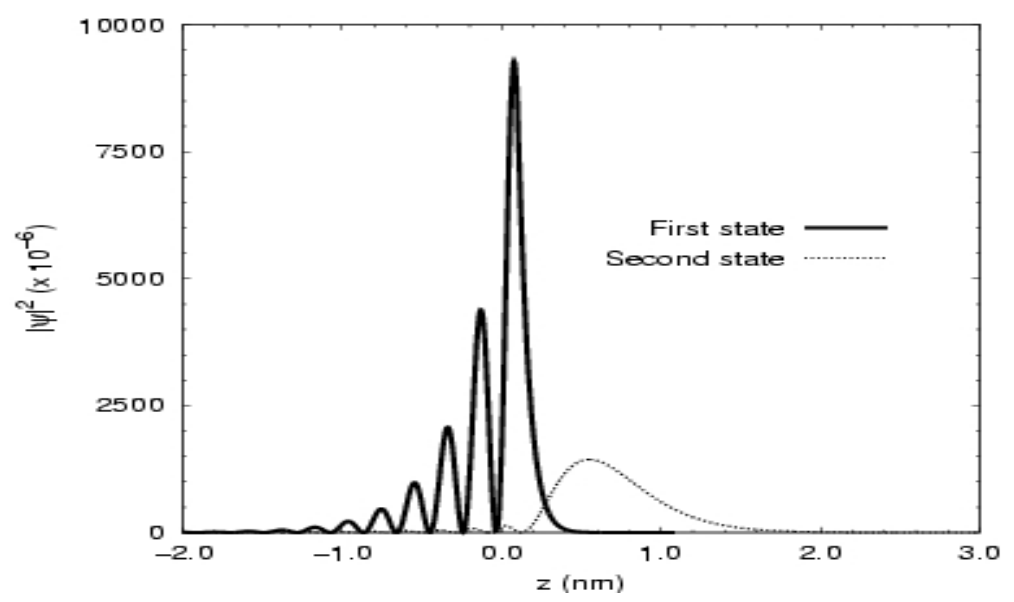

(Fig.3-8) the probability amplitude of the $n=1$ and 2 image states obtained from the image potential calculation, $z=0$ corresponds to the surface atomic layer position.

$\begin{array}{cccc}\text { level } & \text { Energy }(\mathrm{eV}) & \text { Potential } & \text { Gap } \\ 1 & 7,318 & V_{0}=11,25 \mathrm{eV} & E_{\text {midgap }}=8,797 \mathrm{eV} \\ 2 & 10,845 & 2 V_{g}=5 \mathrm{eV} & E_{g b}=6,300 \mathrm{eV} \\ 3 & 11,096 & & E_{g t}=11,293 \mathrm{eV} \\ 4 & 11,164 & & \\ 5 & 11,192 & & \\ 6 & 11,206 & & \\ 7 & 11,214 & & \\ 8 & 11,219 & & \\ 9 & 11,222 & & \\ 10 & 11,225 & & \\ & & & \end{array}$

Tab. 3-4 the Tamm state (level 1) and the nine first image states of the image potential model, because the upper edge of the energy gap lies above the vacuum level there are more surface states. 


\section{Chapter 4}

\section{Two-dimensional lattice electronic structure}

In the previous chapter we obtained a type of wave function that could exist for particular energies in the gaps for a given imaginary $\vec{\kappa}$. These wave functions consist purely of decaying waves in the bulk, and thus, are confined to the surface. The existence of one or more surface states in an energy gap depends on the detailed nature of the potential in the surface region. In this chapter we investigate the electronic structure in direction parallel to the surface. We will investigate the electronic structure of wellordered, clean and stepped $\mathrm{Cu}$ (111) surface as a function of wavevector by means of the scanning tunnelling microscopy.

\subsection{Theoretical descriptions}

The behaviour of a two-dimensional (2D) electron gas is receiving general attention for both fundamental and technological reasons. A 2D electron gas can be found at metalsemiconductor interfaces, in artificially layered semiconductors interfaces, or in some organic charge-transfer salts. The electrons occupying the sp-like surface state band in close-packed noble metal surfaces $(\mathrm{Cu}, \mathrm{Ag}, \mathrm{Au})$ are confined normal to the surface between the vacuum barrier and the crystal band gap. They effectively behave as free electrons in two dimensions, providing a useful ground for testing the properties of the $2 \mathrm{D}$ electron gas. In fact, electron scattering processes at point defects, steps, and isolated atoms result in the formation of standing waves that can be visualized by means of the scanning tunnelling microscope (STM) [Ref10-11].

As a consequence of the 3D periodicity of a bulk solid, electrons eigenstates can be classified by a Bloch wave vector $\vec{k}$. The presence of a surface destroys periodicity in the direction normal to the surface, but periodicity parallel to the surface remains. The electron states in the presence of a surface can thus be characterized by a 2D Bloch wave vector $\vec{k}_{p}$. To calculate the electron movement in direction parallel to the surface we will follow our solution of the Schrödinger equation with the wave function: 


$$
\begin{gathered}
\psi(\vec{r})=C \exp \left(i \vec{k}_{p} \cdot \vec{r}_{p}\right) \cdot \psi_{n}(z), \text { where } \vec{k}_{p}=k_{x} \cdot \hat{x}+k_{y} \cdot \hat{y}, \text { and } \vec{r}_{p}=x \cdot \hat{x}+y \cdot \hat{y} \\
E_{t o t}=E_{n}+E_{p} \Rightarrow E_{t o t}=E_{n}+\hbar^{2} / 2 m\left(k_{x}{ }^{2}+k_{y}{ }^{2}\right)
\end{gathered}
$$

In this wave function, $\psi_{n}(z)$ is defined as (2-10) and describes motion along $z$ axis. In our 2D electron gas model, not only the $E(\vec{k})$ relation but also the density of states is of particular interest.

\subsection{Steps}

We have already seen that the atomic arrangement can be seen by the scanning tunnelling microscopy, STM, (Fig.2-3). By sensing the corrugations in the electron density of the surface, Shockley type surface states have been probed by this method that shows the quantum mechanical probability density distribution of electrons of metals (Fig.3-5). These corrugations in the electron density introduce a well-defined theoretical concept," the Local Density of States". In this context the present work studies the influence of steps on the electron states density at the surfaces. In order to study a sample with well-defined steps I have investigated $\mathrm{Cu}$ (111) surface. Copper is an ideal system that has been studied a lot and its vicinal surface distributes steps regularly. At a vicinal surface the average surface normal is tipped away from a high symmetry low-index direction, and the microscopic structure consists of an ordered array of atomic steps which separate low-index terraces.

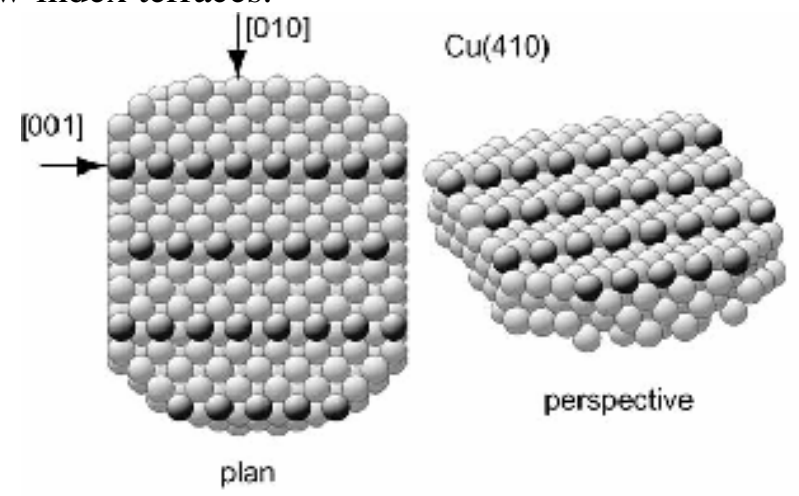

(Fig.4-1) Schematic diagram of ideally terminated $\mathrm{Cu}$ (410) surface in plan and perspective views. The Cu atom row at the down-step edge of each (100) terrace is shown shaded darker for clarity. [Ref.20]

The microscopic atomic structure near a step edge is very different from that found in the bulk or at a low-index surface, and as a consequence one expects a substantial redistribution of the valence electronic charge density near the step edges. This can play an important role in modifying the local dynamical properties and the chemical reactivity. Therefore, stepped surfaces are important systems to study experimentally and theoretically. In fact, the influence of steps on the surface chemistry can be so large that the macroscopic reactivity of many real surfaces is governed by dynamical phenomena at 
steps. Understanding these processes at the microscopic level can lead to control of surface dynamical phenomena through control and design of the surface structure.

Stepped surfaces can be produced by purposely miscutting a crystal forming a vicinal surface. Usually reconstruction will create a surface characterized by wide terraces separated by monatomic steps. In some cases the steps may be two atomic layers high. In extreme cases, height of these steps may become of comparable size to the terraces and the surface forms relatively large facets (areas of low index planes).

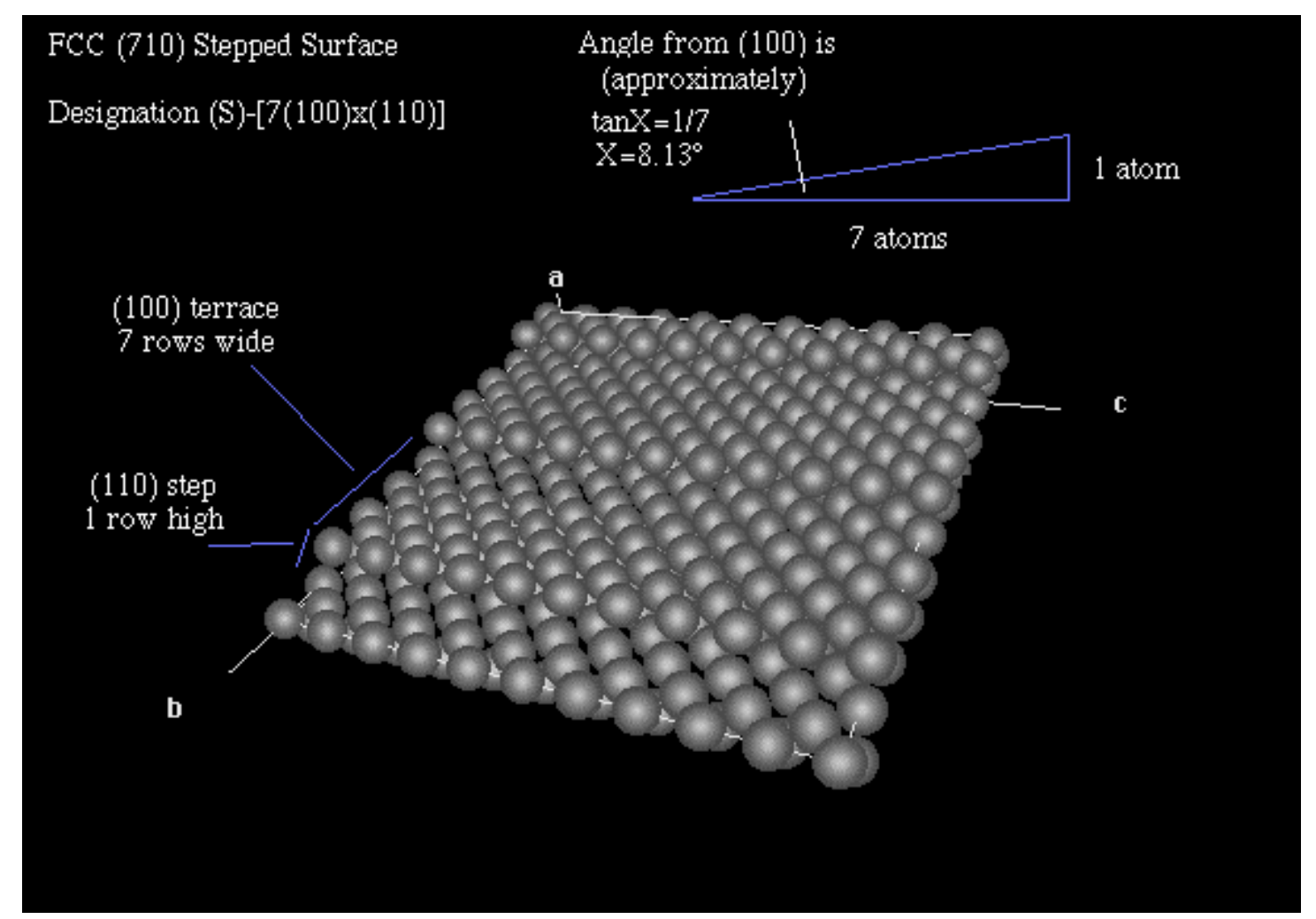

(Fig.4-2)

In the case of the FCC surface shown above (Fig.4-2), the surface is composed of terraces 7 rows wide (including the atoms forming the step edges) which are parallel to the simple low index (100) surface. The steps are 1 layer high and have a (110) structure. Therefore the surface is designated as FCC (S)-[7(100)*(110)] often shortened to $7(100)^{*}(110)$. If the terrace were 10 atoms wide and the step 2 layers high the designation would be (S)-[10(100)*2(110)]. Remember that this is only a model: the real surface will, on average, show this type of structure but locally there will be regions with a range of terrace widths. 


\subsection{Calculation method}

In this essay I have investigated two important questions. First, is there any surface state contained in the band gap and if so, at what energy does it occur? Second, how is the charge distributed? For nearly free electrons (which we consider in this essay) the first question was answered by Shockley for the gaps at the zone boundary (In chapter three, we found out that there is at least one electron state which lies in the middle of the gap). The second question has also been investigated here and we will compare our results to the other theoretical and experimental studies.

\subsubsection{The density of states}

We already know that in the ground state of a system of free electrons, occupied orbitals of the system at $0 K$ fill the Fermi sphere. The number of orbitals per unit energy is called the density of states. This is actually the density of one-particle states or density of orbitals. Density of single-particle states as a function of energy, for free electron gas is shown schematically in Fig.4-3. In three-dimensional free-electron gas the density of states is a function of $\varepsilon^{\frac{1}{2}}$, but it is constant for the two-dimensional case. Now if a threedimensional free-electron gas confined in one dimension and free in the other two, the density of states will be constant between energies that the electrons satisfy the particle in a box conditions in the third dimension. If a two-dimensional electron gas is confined in one dimension, the density of states is on average constant. The peaks appear when the electrons have enough energy to satisfy the conditions of a particle in a box as in first case. Between the peaks the density of states decreases like in one dimensional case i.e. $\varepsilon^{-\frac{1}{2}}$.

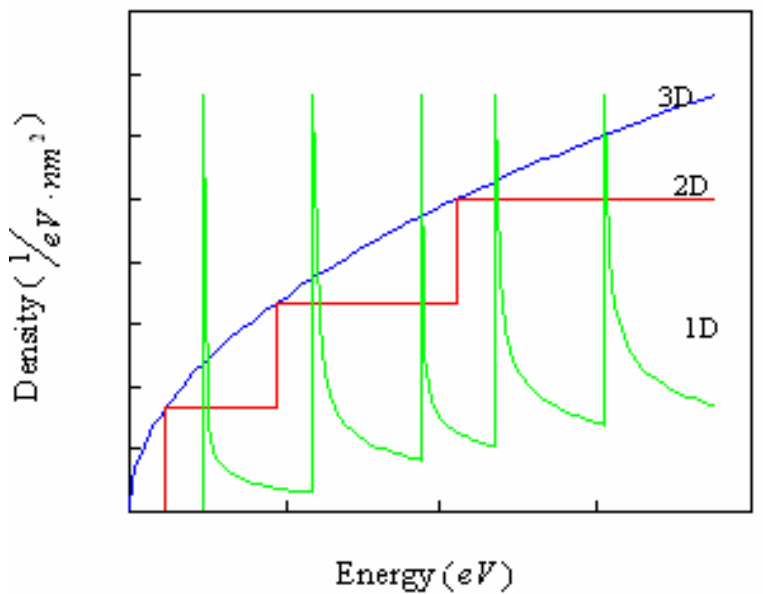

(Fig.4-3) Density of states for free electron gas, for 3D, 2D and $1 D$

The general definition of density of states (DOS) is: 


$$
D(E)=\sum_{n} \delta\left(E-E_{n}\right)
$$

What the above equation tells us is the average number of states over the entire solid. Notice that the density of states changes on an atomic scale. Distribution of eigenfunction amplitudes, $\left|\varphi_{n}(x)\right|^{2}$ characterizes the spatial fluctuations of well defined energy levels. It is simply the total density of states for the system weighted by the squared magnitude of the wave functions in an energy interval at the spatial point in question. The definition of the local density of states (LDOS) in the case of one dimensional free-electron gas is:

$$
D(E, x)=\sum_{n}\left|\varphi_{n}(x)\right|^{2} \delta\left(E-E_{n}\right)
$$

\subsubsection{The Green's functions}

In basic mathematical physics Green's functions play an important role for the solution of linear ordinary and partial differential equation, and are a key component to the development of boundary integral methods [Appendix \& Ref.3]. The Green's function for electrons is defined as:

$$
g\left(E, x, x^{\prime}\right)=\sum_{n} \frac{\varphi_{n}(x) \varphi^{*}{ }_{n}\left(x^{\prime}\right)}{E-E_{n}},
$$

This is a function of energy and distance and is a sum of all the eigenstates. $E$ is the enery variable and $E_{n}$ is the energy of the state $n$. Actually $g\left(E, x, x^{\prime}\right)$ is the amplitude for an electron with energy $E$, released at point $x^{\prime}$, to reach point $x . g\left(E, x, x^{\prime}\right)$ is in general complex. Now if we let $E$ have a tiny imaginary part, $\Delta$, self-energy, we can write:

$$
\begin{aligned}
& \operatorname{Im}\left[g\left(E+i \Delta, x, x^{\prime}\right)\right]=\operatorname{Im}\left[\sum_{n} \frac{\varphi_{n}(x) \varphi_{n}{ }^{*}\left(x^{\prime}\right)}{E+i \Delta-E_{n}}\right] \stackrel{{ }_{x=x^{\prime}}}{\longrightarrow} \\
& =\operatorname{Im}\left[\sum_{n} \frac{\left|\varphi_{n}(x)\right|^{2}}{E+i \Delta-E_{n}}\right]=-\pi \sum_{n}\left|\varphi_{n}(x)\right|^{2} \delta\left(E-E_{n}\right)
\end{aligned}
$$

Note that the Green's functions in our solutions are calculated at $x=x^{\prime}$.

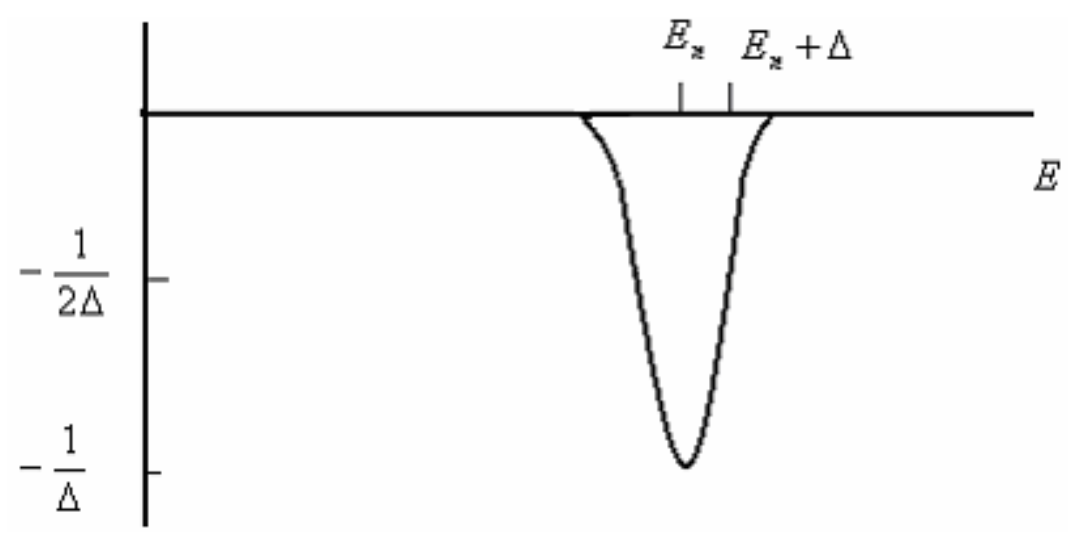

(Fig.4-4) a delta function with imaginary value 
In (4-5), $\left|\varphi_{n}(x)\right|^{2}$ is real and we can show that;

$$
\begin{aligned}
& \operatorname{Im} \frac{1}{E-E_{n}+i \Delta}=\operatorname{Im} \frac{E-E_{n}-i \Delta}{\left(E-E_{n}\right)^{2}+\Delta^{2}}= \\
& \frac{-\Delta}{\left(E-E_{n}\right)^{2}+\Delta^{2}}=-\pi \delta\left(E-E_{n}\right)
\end{aligned}
$$

To prove the last step in (4-6), we look at the integral over energy:

$\int_{-\infty}^{\infty} \frac{-\Delta}{\left(E-E_{n}\right)^{2}+\Delta^{2}} d E \quad \Rightarrow \quad$ there, $\left(E-E_{n}\right)=x \quad \Rightarrow \quad d E=d x$ then;

$\int_{-\infty}^{\infty} \frac{-\Delta}{x^{2}+\Delta^{2}} d x=-\frac{1}{\Delta} \int_{-\infty}^{\infty} \frac{d x}{1+\left(\frac{x}{\Delta}\right)^{2}} \Rightarrow \mid \begin{gathered}t=\frac{x}{\Delta} \\ d x=\frac{\Delta d t}{x}\end{gathered} \Rightarrow$

$-\frac{1}{\Delta} \int_{-\infty}^{\infty} \frac{\Delta d t}{1+t^{2}}=-\int_{-\infty}^{\infty} \frac{d t}{1+t^{2}}=-I_{-\infty}^{\infty} \arctan t=-\pi$

We already know that LDOS is the squared magnitude of the wave functions in a small energy range at the spatial point, then (4-5) becomes;

$$
\operatorname{Im}\left[g\left(E, x, x^{\prime}\right)\right]=-\pi \cdot D(E, x)
$$

Then the local density of states in each point $\left(x=x^{\prime}\right)$ is:

$$
D(E, x)=-\frac{1}{\pi} \cdot \operatorname{Im} g\left(E, x, x^{\prime}\right)
$$

\subsubsection{Equation of motion}

We can deduce an equation of motion for $g$ by letting the (Schrödinger-like) operator $(\hat{H}-E)$ operate on $g$ :

$$
\begin{aligned}
{[\hat{H}-E] g\left(E, x, x^{\prime}\right) } & =[\hat{H}-E] \sum_{n} \frac{\varphi_{n}(x) \varphi_{n}{ }^{*}\left(x^{\prime}\right)}{E-E_{n}} \\
& =\sum_{n} \frac{\left(E_{n}-E\right) \varphi_{n}(x) \varphi_{n}{ }^{*}\left(x^{\prime}\right)}{E-E_{n}}=-\sum_{n} \varphi_{n}(x) \varphi_{n}{ }^{*}\left(x^{\prime}\right)
\end{aligned}
$$

The right hand side in (4-10) is a Dirac delta function. To show that, there is a general theorem in quantum mechanic that states: an arbitrary function $\varphi_{n}(x)$ can be expanded in a complete set of eigenfunctions of Hamiltonian, that is [Ref.4],

$$
f(x)=\sum_{n} f_{n} \varphi_{n}(x),
$$


If we choose the eigenfunctions of Hamiltonian to be normalized, and if we take into account that the eigenfunctions are orthogonal, the expansion coefficients can be determined by:

$$
f_{n}=\int_{-\infty}^{+\infty} \varphi_{n}^{*}(x) f(x) d x
$$

We take:

$$
f(x)=\delta\left(x-x^{\prime}\right)
$$

Then the expansion coefficients,

$$
f_{n}=\int_{-\infty}^{\infty} \varphi_{n}^{*}(x) \delta\left(x-x^{\prime}\right) d x=\varphi_{n}^{*}\left(x^{\prime}\right),
$$

Then (4-11) gives:

$$
\delta\left(x-x^{\prime}\right)=\sum_{n} \varphi_{n}^{*}\left(x^{\prime}\right) \varphi_{n}(x)
$$

Now with comparing (4-9) and (4-14) and for any point but $x^{\prime},\left(x \neq x^{\prime}\right)$

$$
(\hat{H}-E) g\left(E, x, x^{\prime}\right)=0
$$

Then, the most general solution is:

$$
g^{ \pm}\left(E, x, x^{\prime}\right)=C^{ \pm} e^{ \pm i k x}
$$

These correspond to outgoing waves in both directions from $x^{\prime}$. At this point, $x^{\prime}, g$ must be continuous but its derivative is not because of a cusp at $x=x^{\prime}$ that make matching process difficult. At this point and for the incoming waves,

$$
\begin{aligned}
& \text { 1. }\left.g^{+}\left(E, x, x^{\prime}\right)\right|_{x=x^{\prime}}=\left.g^{-}\left(E, x, x^{\prime}\right)\right|_{x=x^{\prime}} \\
& \text { 2. } \int_{x^{\prime}-\delta}^{x^{\prime}+\delta}(\hat{H}-E) g\left(E, x, x^{\prime}\right) d x=\int_{x^{\prime}-\delta}^{x^{\prime}+\delta}-\delta\left(x-x^{\prime}\right) d x=-1
\end{aligned}
$$

This means:

2a. $\int_{x^{\prime}-\delta}^{x^{\prime}+\delta}\left[-\frac{\hbar^{2}}{2 m} \frac{d^{2}}{d x^{2}}+V(x)\right] g\left(E, x, x^{\prime}\right)-\int_{x^{\prime}-\delta}^{x^{\prime}+\delta} E g\left(E, x, x^{\prime}\right)=-1$

$-\left.\frac{\hbar^{2}}{2 m} \frac{d g\left(E, x, x^{\prime}\right)}{d x}\right|_{x^{\prime}-\delta} ^{x^{\prime}+\delta}=-\frac{\hbar^{2}}{2 m}\left[\left(\frac{d g\left(E, x, x^{\prime}\right)}{d x} \mid x=x^{\prime+}\right)-\left(\frac{d g\left(E, x, x^{\prime}\right)}{d x} \mid x=x^{\prime-}\right)\right]=-1$

$\frac{\hbar^{2}}{2 m}\left(\left.\frac{d g}{d x}\right|_{+}-\left.\frac{d g}{d x}\right|_{-}\right)=\left.1 \Rightarrow \frac{d g}{d x}\right|_{+}-\left.\frac{d g}{d x}\right|_{-}=\frac{2 m}{\hbar^{2}}$

With these two conditions, (4-17) and (4-18), we can find the eigenfunctions for our onedimensional problem;

$$
\begin{gathered}
C^{+}=\frac{-i m}{k_{n} \hbar^{2}} e^{i k x^{\prime}} \quad \& \quad C^{-}=\frac{-i m}{k_{n} \hbar^{2}} e^{-i k x^{\prime}} \\
g\left(E, x, x^{\prime}\right)=\frac{-i m}{k \hbar^{2}} \exp \left(i k\left|x-x^{\prime}\right|\right)
\end{gathered}
$$


Using (4-19) in (4-8), shows that the density of states is proportional to the inverse wave vector and thus, proportional to $\frac{1}{\sqrt{E}}$, which agrees with our expectation of one dimensional electron gas from earlier [Ref.2];

$$
\begin{aligned}
& D(E, x)=-\frac{1}{\pi} \cdot \operatorname{Im} g\left(E, x, x^{\prime}\right) \quad \Rightarrow \\
& D(E, x)=-\frac{1}{\pi} \cdot \operatorname{Im}\left[\frac{-i m}{k \hbar^{2}} \exp \left(i k\left|x-x^{\prime}\right|\right)\right] \stackrel{x=x^{\prime}}{\longrightarrow} \\
& D(E, x)=-\frac{1}{\pi} \cdot \operatorname{Im}\left[\frac{-i m}{k \hbar^{2}}\right]
\end{aligned}
$$

\subsection{Green's functions for two-dimensional terrace}

The eigenfunctions problem for the electrons between edges at a terrace, that we already considered as hard walls will be similar to the problem for a particle in a box. This implies that the wave functions must vanish for $y \leq 0$ and $y \geq a$ with $a$ being the terrace width. The solutions of the Schrödinger equation in this case are $\sin k x$ and $\cos k x$. The boundary conditions imply that:

$$
k x=n \pi \quad n=1,2,3 \ldots
$$

The normalized sine solution is:

$$
\varphi_{n}(y)=\sqrt{\frac{2}{a}} \sin \left(\frac{n \pi y}{a}\right)
$$

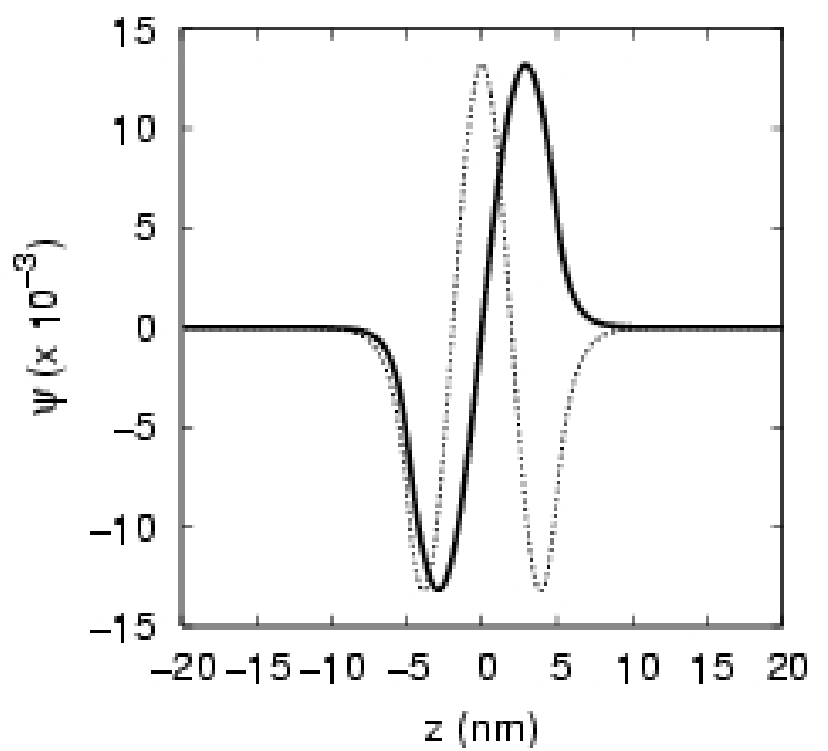

(Fig.4-5) Eigensolutions for particle in a box. $(n=1$ and $n=2)$ 
It turns out that the Green's function for the two-dimensional terrace can be written as:

$$
\begin{gathered}
G\left(x-x^{\prime}, y, y^{\prime}, E\right)=\sum_{n} g_{n}\left(x-x^{\prime}, E\right) \cdot \sin \left(\frac{n \pi y}{a}\right) \cdot \sin \left(\frac{n \pi y^{\prime}}{a}\right), \\
g_{n}\left(x-x^{\prime}, E\right)=g_{n}\left(x-x^{\prime}, E-\frac{n^{2} \pi^{2} \hbar^{2}}{2 m a^{2}}\right)
\end{gathered}
$$

Here $E-\frac{n^{2} \pi^{2} \hbar^{2}}{2 m a^{2}}$ is the energy left for the motion along the $x$ direction.Then the Schrödinger equation with this function that contains all variables can be written:

$$
[\widehat{H}(x, y)-E] G\left(x-x^{\prime}, y, y^{\prime}, E\right)=-\delta\left(x-x^{\prime}\right) \cdot \delta\left(y-y^{\prime}\right),
$$

Where:

$$
\hat{H}=-\frac{\hbar^{2}}{2 m}\left(\partial^{2} / \partial x^{2}+\partial^{2} / \partial y^{2}\right)+V
$$

In this case the Green's function, $G\left(x-x^{\prime}, y, y^{\prime}, E\right)$ is a function of both $x$ and $y$. To get the density of states from the Green's function we set $x=x^{\prime}$ and $y=y^{\prime}$ as a consequence, the local density of states becomes a function of its position at the terrace, $y$ but it is independent of $x$ (a function of $E$ and $y$ ). The local density of states turns out to be a function of the position at the terrace in a small range at the spatial point;

$$
G\left(x-x^{\prime}, y, y^{\prime}, E\right) \stackrel{x=x^{\prime} \& y=y^{\prime}}{\longrightarrow} D(y, E),
$$

In this context, there is another factor that affects the shape of the local density of states drastically, namely $\tau$, the lifetime. Lifetime is defined as the time between two collisions that an electron collides with other electrons and loses its energy until it falls down to the bulk states. It is the similar for an excited electron when a photon is absorbed. The electron goes up to an excited state but it goes down eventually after releasing a photon. The time that electron spends at this excited state is also called lifetime. These two definitions are actually the same because both are about the time an electron can be in an excited state. The potential energy that we consider is an imaginary potential, can be defined as;

$$
\begin{aligned}
& V=-i \Delta, \text { then; } \\
& E-V=E+i \Delta,
\end{aligned}
$$

Then the equation for $G\left(x-x^{\prime}, y, y^{\prime}, E\right)$ with $\Delta$ absorbed in $E(E \rightarrow E+i \Delta)$ becomes:

$$
\begin{aligned}
& \sum_{n}\left[-\frac{\hbar^{2}}{2 m} \cdot \frac{\partial^{2} g_{n}\left(x-x^{\prime}, E\right)}{\partial x^{2}}-\left(\frac{\hbar^{2} n^{2} \pi^{2}}{2 m a^{2}}+E\right) g_{n}\left(x-x^{\prime}, E\right)\right] \sin \left(\frac{n \pi y}{a}\right) \sin \left(\frac{n \pi y^{\prime}}{a}\right)=0 \\
& \Rightarrow \quad \frac{\partial^{2}}{\partial x^{2}} g_{n}\left(x-x^{\prime}, E\right)+\left[\frac{2 m E}{\hbar^{2}}-\frac{n^{2} \pi^{2}}{a^{2}}\right] g_{n}\left(x-x^{\prime}, E\right)=0
\end{aligned}
$$

The solution, as we have seen before is: 
$g_{n}\left(x-x^{\prime}, E\right)=C_{n} \exp \left[i k_{n}\left|x-x^{\prime}\right|\right]$

Where, $k_{n}{ }^{2}=\left(k_{1}{ }^{2}-k_{2}{ }^{2}\right)=\frac{2 m E}{\hbar^{2}}-\frac{n^{2} \pi^{2}}{a^{2}} \Rightarrow k_{n}=\sqrt{\left(\frac{2 m E}{\hbar^{2}}-\frac{n^{2} \pi^{2}}{a^{2}}\right)}$

1. $E\rangle \frac{n^{2} \pi^{2} \hbar^{2}}{2 m a^{2}} \quad \Rightarrow \quad k_{n}$ is real and $g$ is a propagating plane wave along the step.

2. $E<\frac{n^{2} \pi^{2} \hbar^{2}}{2 m a^{2}} \quad \Rightarrow \quad k_{n}$ is imaginary and $g$ decrease exponentially along the step.

The wave function is continuous at $x=x^{\prime}$, but not its derivative. This is the same solution we provided for the one dimensional case. Then the solution for the $2 \mathrm{D}$ electron gas is:

$G_{n}\left(x-x^{\prime}, y, y^{\prime}, E\right)=\sum_{n}\left(\frac{-i m}{k_{n} \hbar^{2}} e^{i k_{n}\left|x-x^{\prime}\right|}\right) \cdot \sin \left(\frac{n \pi y}{a}\right) \cdot \sin \left(\frac{n \pi y^{\prime}}{a}\right)$

Now we can develop our expression for the local density of states (4-9), for the 2D electron gas:

$$
\begin{aligned}
& D(E, x, y)=- \frac{1}{\pi} \operatorname{Im} G_{n}\left(x-x^{\prime}, y, y^{\prime}, E\right) \Rightarrow \\
& D(E, x, y)=-\frac{1}{\pi} \operatorname{Im}\left[\sum_{n}\left(\frac{-i m}{k_{n} \hbar^{2}} e^{i k_{n}\left|x-x^{\prime}\right|}\right) \cdot \sin \left(\frac{n \pi y}{a}\right) \cdot \sin \left(\frac{n \pi y^{\prime}}{a}\right)\right] \quad \underset{x=x^{\prime} \& y=y^{\prime}}{\longrightarrow} \\
& D(E, x, y)=-\frac{1}{\pi} \operatorname{Im}\left[\sum_{n} \frac{-i m}{k_{n} \hbar^{2}} \cdot \sin ^{2}\left(\frac{n \pi y}{a}\right)\right]
\end{aligned}
$$

In our solution we considered a terrace i.e. we contained the electron in a box with length $a$. The system is two dimensional but in this case we have confined the electron in one direction, it is the reason why we do not get a constant density of states. In following section we will discuss the origin of these peaks. Fig (4-6) shows a plot of the local density of states, (4-35) at the stepped $\mathrm{Cu}$ (111) surface with terrace width $50 \AA$.

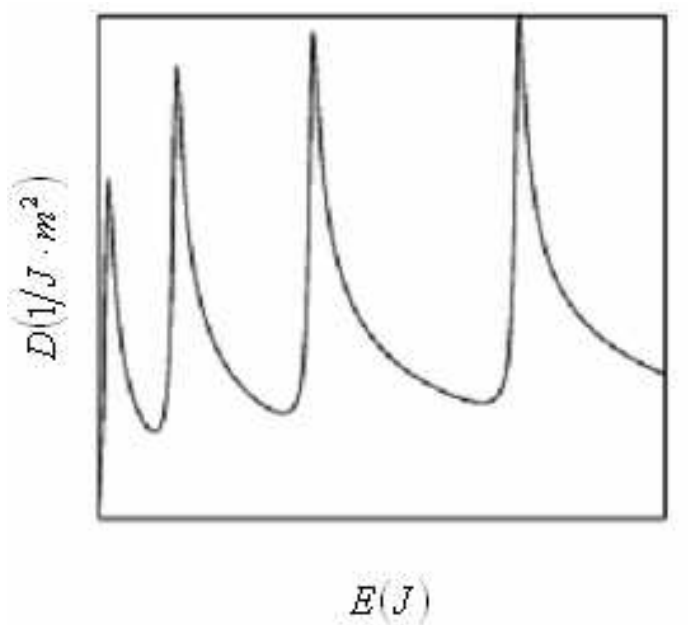

(Fig.4-6) Plot of the local density of states (eqv. (4-35)). 


\subsection{Results and discussions}

In our investigation of electronic structure of stepped surfaces, we began with a series of approximations and put some conditions to simplify our problem in a sensible way. Nearly-free and free electron approximation resulted in shape of the band theory and the free electron gas model for the electrons at the surface. With these approximations and conditions like clean and well-ordered surfaces, the solution of Schrödinger-like Green's function equation, (4-27) gave a two-dimensional wave function (4-33). Then we were able to find the density of states for this two-dimensional case.

For a three dimensional free-electron gas that is confined in two dimensions the density of states is a step function with steps occurring at the energy of each quantized level for the third dimension (Fig.4-3). In our case, on the other hand, the system (each step at the surface) represents a two dimensional free-electron gas that is confined in one dimension therefore the density of states must be an the average constant with peaks at the energy of each quantized level for the other dimension (Fig.4-6).

Equation (4-34) gives the local density of states at a terrace of a $\mathrm{Cu}$ (111) surface. It means the local density of states (LDOS) for each vicinal surface with terraces at this direction. Figure (4-7) shows a plot of LDOS for a terrace with width $50 \AA$ in direction (111) but different lifetimes. It does not look like the plot of density of states for the 2D electron gas, but it is approximately the same. We have already seen that the local density of states for our case, terrace with hard walls, is an average constant function of energy and the peaks appear when the electrons satisfy the boundary conditions for a particle in a box, i.e. energies associated with the quantized level. From (4-35) one can see that the peaks appear for odd values of $n\left(n^{2}=1,9,25,49\right)$.

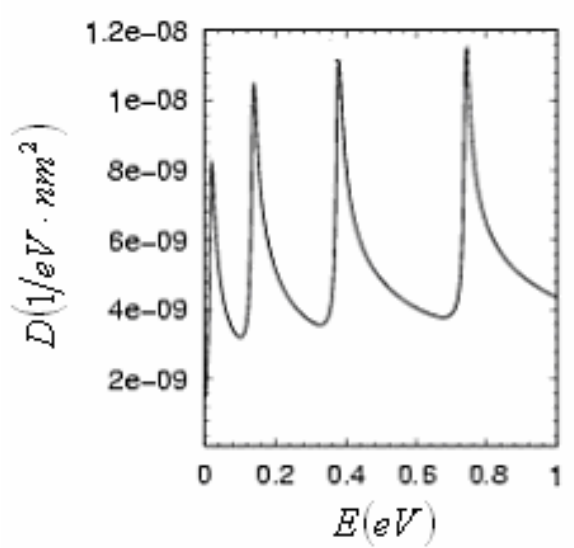

(a)

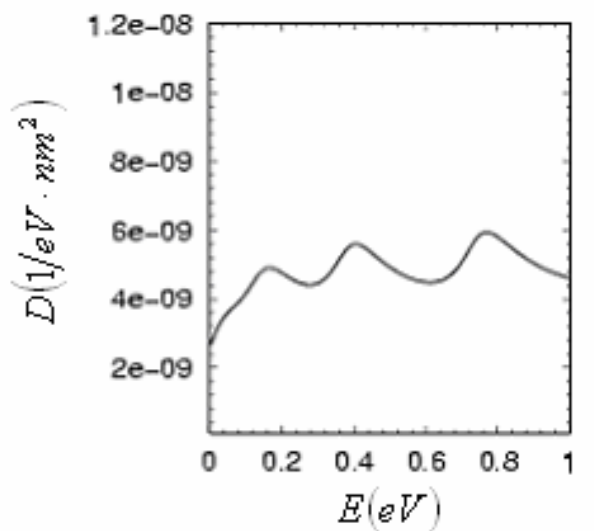

(b)

(Fig.4-7) density of states associated with stepped $\mathrm{Cu}$ (111) surfaces at the middle of the terrace with lifetime, $(a) ; \tau=100 \mathrm{fs}$ and $(b) ; \tau=10 \mathrm{fs}$ 
The electrons with energies of the peaks are able to satisfy the conditions for particle in a box in direction perpendicular to the steps and also the conditions for onedimensional electron gas at the other direction. For energies between the peaks, the local density of states decreases just like the one-dimensional case. Fig.4-6 shows the lifetimedependence of the local density of states from $\mathrm{Cu}$ (111). The longer the lifetime, the higher the peaks in LDOS diagram.

Photo-electron spectroscopy is a very powerful tool for investigation of the three dimensional bulk as well as the two dimensional surface bands. Not only the $E(\vec{k})$ relation but also the lifetimes of the excited electronic states are of particular interest. The lifetime is of interest as it determines the mean free path of the surface state electrons, and hence the effective range of surface state mediated interactions. This can be determined by line width analysis in photoemission spectra. These studies of line widths and line shapes contain important information of lifetime broadening of the final hole and electron states, on electron-hole pair excitations and conduction-electron screening in the bulk and at the surface. The effects of the lifetime on Shockley surface state electron can also be measured by the scanning tunnelling microscopy, STM. Using low-temperature STM spectroscopy one can calculate the relation between the geometrical width of the onset and the imaginary self-energy $(\Sigma=\hbar / 2 \tau=\Delta / 2$ ). In general, the natural line width is the inverse lifetime of the photohole. A number of investigations with photoemission spectra have provided an important picture of the relations between the local density of states, lifetime and the vicinal faces width. All these investigations lead to that the peaks width for the vicinal surfaces increases compared to the peak width of the unstepped surfaces and this increasing is actually of the same order as the lifetime width [Ref.1215]. This is illustrated in Fig.4-7. 


\section{Summary}

Summarizing I can state that the calculations in this paper yield an understanding of the surface electronic states and the electronic structure of vicinal faces of $\mathrm{Cu}(111)$ by the means of the scanning tunneling microscopy (STM).

For an idealized one-dimensional crystal it is possible to have energy levels whose wave functions are localized at the surface. These states are called surface states. There is one surface state for each energy gap between the ordinary allowed bands of energies. These electron states are called Tamm states. This Tamm state has an energy that lies almost at the middle of the energy gap and is mainly localized at the surface atomic layer. The image potential states are generated by a potential well formed by the Coulomb-like image potential barrier. These image states that are also called Shockley states are localized in a slowly decaying tail in the vacuum.

I also studied the lateral (in-plane) motion of electrons confined to terraces between steps on a vicinal $\mathrm{Cu}$ (111) surface. The local density of states showed a number of peaks at energies where electrons can occupy new quantum-well states on a step. I also tested the influence of the electron lifetime on the local density of states.

Acknowledgements: I am grateful to my teacher and supervisor Dr. Peter Johansson for many discussions on the subject of solid state physics and critical comments on various aspects of this work. I would also like to thank the examiner, Dr. Andreas Oberstedt. 


\section{Appendix A: Introduction to Green's Functions}

Green's function is a basic solution to a linear differential equation, a building block that can be used to construct many useful solutions. For heat conduction, for example, the Green's Function is proportional to the temperature caused by a concentrated energy source. The exact form of the Green's Function depends on the differential equation, the body shape, and the type of boundary conditions present. Green's functions are named in honour of English mathematician and physicist George Green (1793-1841). Green's functions play an important role in the solution of linear ordinary and partial differential equations, and are a key component to the development of boundary integral equation methods.

Consider a linear differential equation written in the general form

$$
L(x) u(x)=f(x)
$$

where $\mathrm{L}(\mathrm{x})$ is a linear, self-adjoint differential operator, $u(x)$ is the unknown function, and $f(x)$ is a known non-homogeneous term. Operationally, we can write a solution to equation (1) as

$$
u(x)=L^{-1}(x) f(x)
$$

where $L^{-1}$ is the inverse of the differential operator $L$. Since $L$ is a differential operator, it is reasonable to expect its inverse to be an integral operator. We expect the usual properties of inverses to hold,

$$
L L^{-1}=L^{-1} L=I
$$

where $I$ is the identity operator. More specifically, we define the inverse operator as

$$
L^{-1} f=\int G\left(x, x^{\prime}\right) f\left(x^{\prime}\right) d x^{\prime}
$$

where the kernel $G\left(x, x^{\prime}\right)$ is the Green's Function associated with the differential operator $L$. Note that $G\left(x, x^{\prime}\right)$ is a two-point function which depends on both $x$ and $x^{\prime}$. To complete the idea of the inverse operator $L$, we introduce the Dirac delta function as the identity operator $I$. Recall the properties of the Dirac delta function $\delta(x)$ are 


$$
\begin{aligned}
& \int_{-\infty}^{\infty} \delta\left(x-x^{\prime}\right) f\left(x^{\prime}\right) d x^{\prime}=f(x) \\
& \int_{-\infty}^{\infty} \delta\left(x^{\prime}\right) d x^{\prime}=1
\end{aligned}
$$

The Green's function $G\left(x, x^{\prime}\right)$ then satisfies

$$
L(x) G\left(x, x^{\prime}\right)=\delta\left(x-x^{\prime}\right)
$$

The solution to equation (1) can then be written directly in terms of the Green's function as

$$
u(x)=\int_{-\infty}^{\infty} G\left(x, x^{\prime}\right) f\left(x^{\prime}\right) d x^{\prime}
$$

To prove that equation (7) is indeed a solution to equation (1), simply substitute as follows:

$$
\begin{aligned}
L u(x) & =L \int_{-\infty}^{\infty} G\left(x, x^{\prime}\right) f\left(x^{\prime}\right) d x^{\prime} \\
& =\int_{-\infty}^{\infty} L G\left(x, x^{\prime}\right) f\left(x^{\prime}\right) d x^{\prime} \\
& =\int_{-\infty}^{\infty} \delta\left(x-x^{\prime}\right) f\left(x^{\prime}\right) d x^{\prime} \\
& =f(x)
\end{aligned}
$$

Note that we have used the linearity of the differential and inverse operators in addition to equations (4), (5), and (6) to arrive at the final answer. 


\section{Appendix B: Listing of programs used for the numerical calculations}

\section{Calculations of the surface states in one-dimensional band theory}

The main program, "test", is actually the program that uses all the calculated values in subroutines, functions and modules to identify the boundary conditions, energy levels, shape of the wave functions in different positions and derive the wave functions associated with these energies. To derive the wave functions we need the wave vector, $\vec{k}$ and the phase shift, $\delta$. These are both variables of energy and are calculated in the module "kappa_mod". It is important to know that this program picks up just the imaginary values of the wave vector which we are most interested. Module "det_mod" calls these calculated values and uses them to solve the "central equation", (3-7) for both the bulk and the vacuum as well. For the vacuum part, we have two different form of potential. For the image potential model which must be solved numerically, there are three programs to do that. In modules "ystat_mod" and "yimage_mod" a series of vectors are made with the initial values of the wave function, its derivative, energy and normalized value of the wave function. These vectors are known after the numerical solution in module "odeint_mod" and finally in module "imderiv_mod". Now we have calculated all values we need to derive the wave functions back in the program "test".

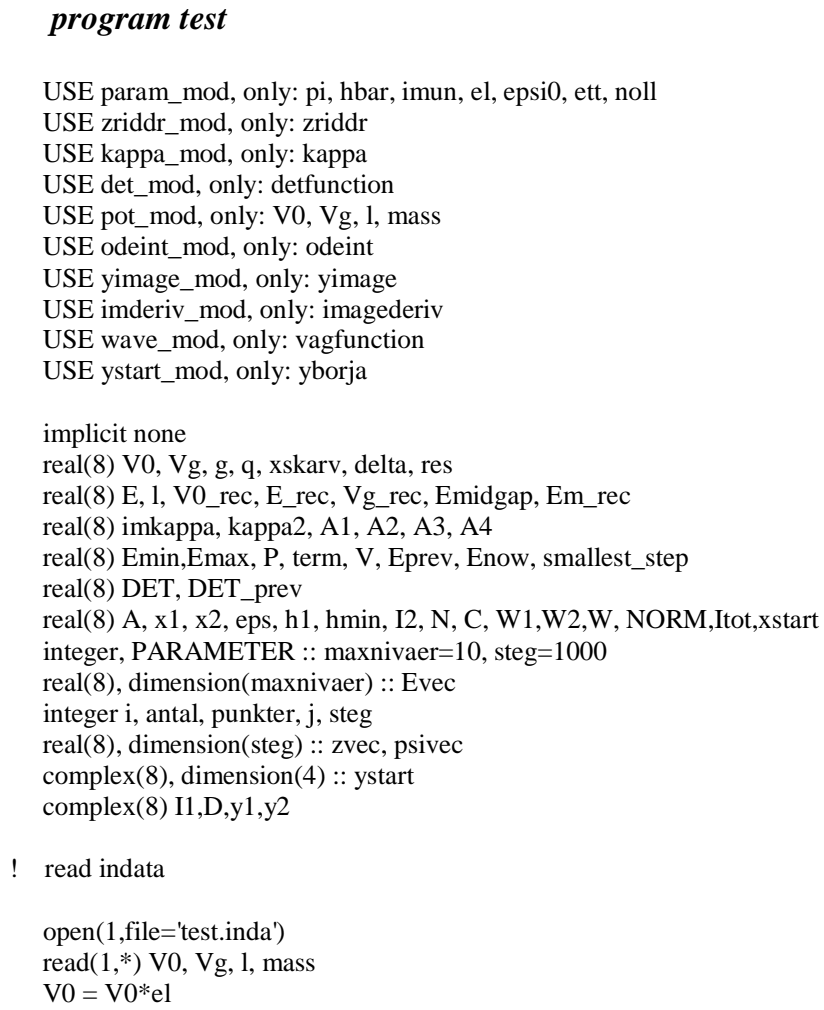




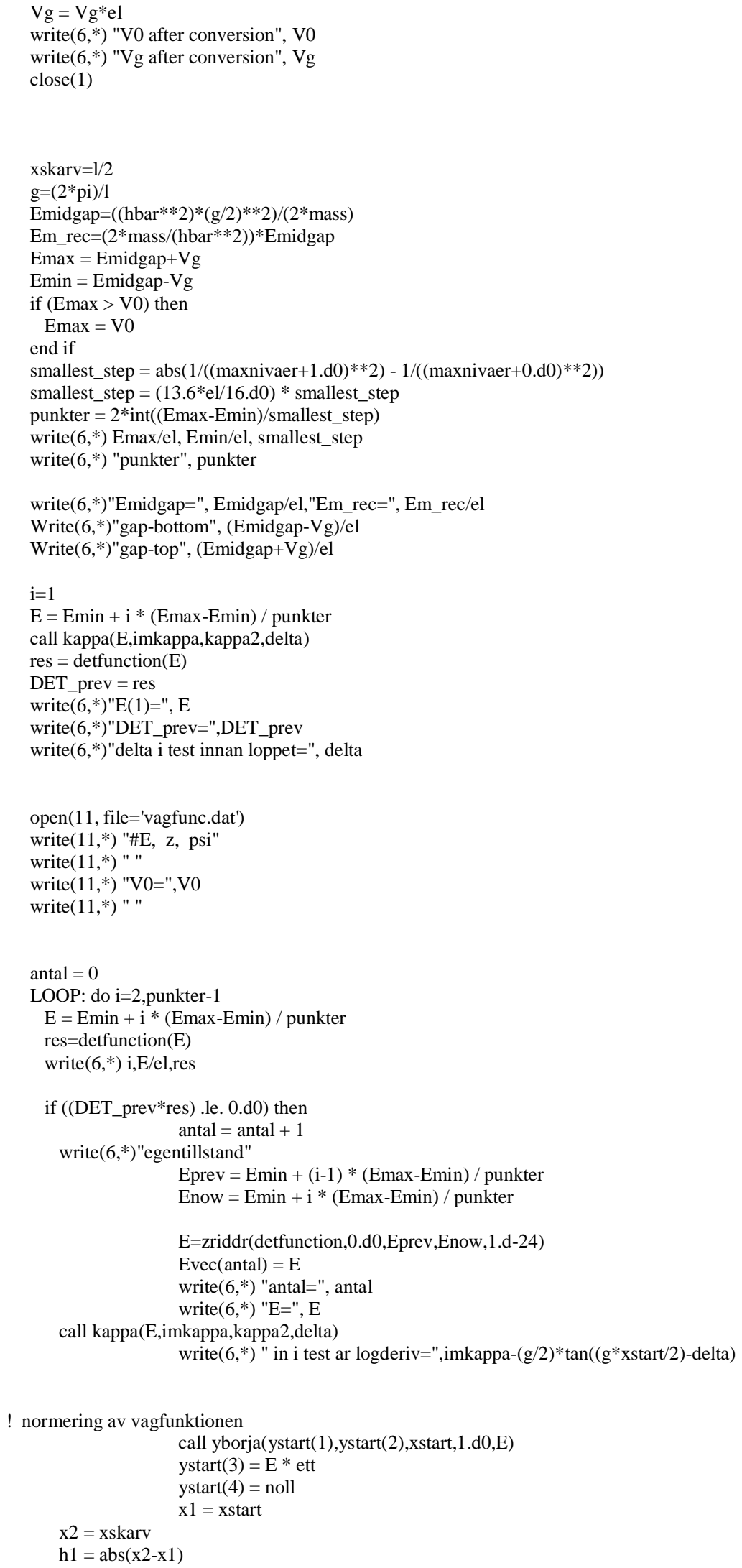




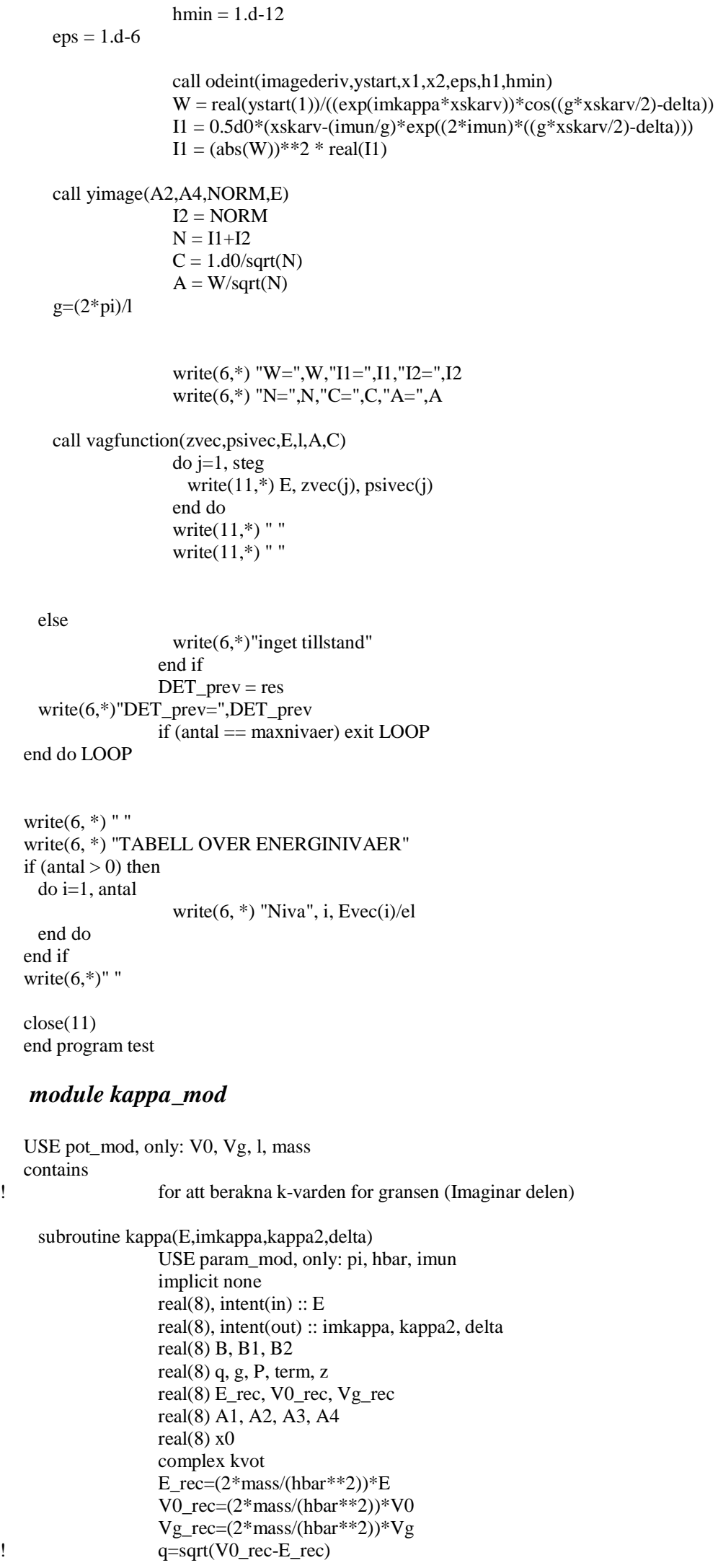

module kappa_mod

USE pot_mod, only: V0, Vg, 1 , mass contains for att berakna k-varden for gransen (Imaginar delen)

subroutine kappa(E,imkappa,kappa2,delta)

USE param_mod, only: pi, hbar, imun implicit none

real(8), intent(in) :: $\mathrm{E}$

real(8), intent(out) :: imkappa, kappa2, delta

$\operatorname{real}(8) \mathrm{B}, \mathrm{B} 1, \mathrm{~B} 2$

real(8) q, g, P, term, z

real(8) E_rec, V0_rec, Vg_rec

real(8) A1, A2, A3, A4

$\operatorname{real}(8) \times 0$

complex kvot

E_rec $=(2 * \operatorname{mass} /($ hbar**2) $) * \mathrm{E}$

V0 rec $=(2 * \operatorname{mass} /(\mathrm{hbar} * * 2)) * \mathrm{~V} 0$

$\mathrm{Vg} \_$rec $=(2 *$ mass $/($ hbar $* * 2)) * \mathrm{Vg}$

! q=sqrt(V0_rec-E_rec) 
$\mathrm{g}=(2 * \mathrm{pi}) / \mathrm{l}$

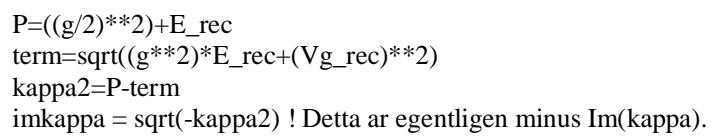

! slut pa att rakna imkappa, borjar med determinanten.

kvot $=\operatorname{sqrt}(($ E_rec-kappa2-((g/2)**2)+(imun*imkappa*g) $) /($ Vg_rec $))$

write $(6, *) " k v o t="$, kvot

delta $=\operatorname{atan} 2(\operatorname{aimag}(k v o t)$, real $(k v o t))$

write $(6, *)$ "delta i kappa_mod=", E/1.60219d-19, delta

end subroutine kappa

! SLUT PA Det har har att gora med determinanten...

end module kappa_mod

\section{module det mod}

USE pot_mod, only: $\mathrm{V} 0, \mathrm{Vg}, 1$, mass contains

function detfunction(E) result(res)

USE param_mod, only: pi, hbar,imun

USE kappa_mod, only: kappa, $\mathrm{V} 0, \mathrm{Vg}, 1$, mass

USE yimage_mod, only: yimage

USE imderiv_mod, only: imagederiv

USE odeint_mod, only: odeint

implicit none

real(8), intent(in) $:: \mathrm{E}$

real(8) res, NORM

real(8) imkappa, kappa2, delta, A1, A2, A3, A4

real(8) g, Vg_rec, V0_rec, E_rec, $x 0$, q

$\operatorname{real}(8) \mathrm{x}, \mathrm{x} 1, \mathrm{x} 2, \mathrm{~h} 1, \mathrm{hmin}, \mathrm{eps}$

complex(8), dimension(4) :: ystart

complex(8) kvot

$\mathrm{g}=(2 * \mathrm{pi}) / \mathrm{l}$

$\mathrm{x} 0=1 / 2 . \mathrm{d} 0$

E_rec $=(2 *$ mass $/($ hbar $* 2)) * \mathrm{E}$

V0_rec $=(2 * \operatorname{mass} /(\mathrm{hbar} * * 2)) * \mathrm{~V} 0$

Vg_rec $=(2 * \operatorname{mass} /(\mathrm{hbar} * * 2)) * \mathrm{Vg}$

call kappa(E,imkappa,kappa2,delta)

$\mathrm{A} 1=(\exp (\mathrm{imkappa} * \mathrm{x} 0)) * \cos ((\mathrm{g} / 2) * \mathrm{x} 0)$-delta $)$

A3 $=$ imkappa*exp(imkappa*x0)* $\cos (((\mathrm{g} / 2) * \mathrm{x} 0)$-delta $)$

$\mathrm{A} 3=\mathrm{A} 3-(\mathrm{g} / 2 . \mathrm{d} 0) * \exp ($ imkappa* $\mathrm{x} 0) * \sin ((\mathrm{g} / 2) * \mathrm{x} 0)-$ delta $)$

! This is for a square barrier, for now.

$\mathrm{q}=\mathrm{sqrt}\left(\mathrm{V} 0 \_\right.$rec-E_rec $)$

$\mathrm{A} 2=-\exp (-\mathrm{q} * \mathrm{x} 0)$

! $\mathrm{A} 4=\mathrm{q} * \exp (-\mathrm{q} * \mathrm{x} 0)$

! This is for an imaginary barrier.

call yimage(A2, A4, NORM, E)

res $=(\mathrm{A} 1 * \mathrm{~A} 4)-(\mathrm{A} 2 * \mathrm{~A} 3)$

write $(6, *) " \mathrm{~A} 1=", \mathrm{~A} 1$, "A2=",A2

write $(6, *) " \mathrm{~A} 3=", \mathrm{~A} 3$, ,A4=",A4

end function detfunction

end module det_mod 
module yimage_mod

contains

subroutine yimage(A2, A4, NORM, E)

USE param_mod, only: pi, el, epsi0, imun, noll, ett, hbar

USE pot_mod, only: V0,Vg,l, mass, zimage

USE imderiv_mod, only: imagederiv

USE ystart_mod, only: yborja

USE odeint_mod, only: odeint

implicit none

real(8), intent(out) :: A2, A4, NORM

real(8), intent(in) $:: \mathrm{E}$

$\operatorname{real}(8) \mathrm{h} 1, \mathrm{hmin}, \mathrm{x} 1, \mathrm{x} 2$

real(8) eps

complex(8) y1, y2

complex(8), dimension(4):: ystart

$\mathrm{x} 2=1 / 2$

call yborja $(y 1, y 2, x 1,1 . d 0, E)$

$\mathrm{ystart}(1)=\mathrm{y} 1$

$y \operatorname{start}(2)=y 2$

ystart $(3)=\mathrm{E} *$ ett

ystart $(4)=$ noll

eps $=1 . d-6$

$\mathrm{h} 1=\operatorname{abs}(\mathrm{x} 1-\mathrm{x} 2)$

$\mathrm{hmin}=1 . \mathrm{d}-12$

call odeint(imagederiv,ystart,x1,x2,eps, h1,hmin)

$\mathrm{A} 2=\operatorname{real}(\operatorname{ystart}(1))$

$\mathrm{A} 4=\operatorname{real}(\mathrm{ystart}(2))$

$\mathrm{NORM}=\operatorname{real}(\mathrm{ystart}(4))$

write $(6, *)$ " in i yimage ar logderv=", A4/A2, real(ystart(2))/real(ystart(1))

end subroutine yimage

end module yimage_mod

\section{module ystart_mod}

contains

subroutine yborja(y1,y2, xstart,C,E)

USE param_mod, only: pi, el, epsi0, imun, noll, ett, hbar

USE pot_mod, only: V0,Vg, 1 , mass, zimage

USE imderiv_mod, only: imagederiv

USE odeint_mod, only: odeint

implicit none

complex(8), intent(out) :: y1, y2

real(8), intent(out) :: xstart

real(8), intent(in) :: E,C

$\operatorname{real}(8)$ zstart, h1, hmin, $\mathrm{x} 1, \mathrm{x} 2, \mathrm{x} 0$

real(8) V_rec, E_rec, V, q, eps

$\operatorname{real}(8)$ keff, zeff

$\operatorname{keff}=\operatorname{sqrt}\left(\left(2 *\right.\right.$ mass $/\left(\right.$ hbar $\left.\left.\left.^{* * 2}\right)\right) * \operatorname{abs}(\mathrm{V} 0-\mathrm{E})\right)$

zeff $=1 /$ keff 


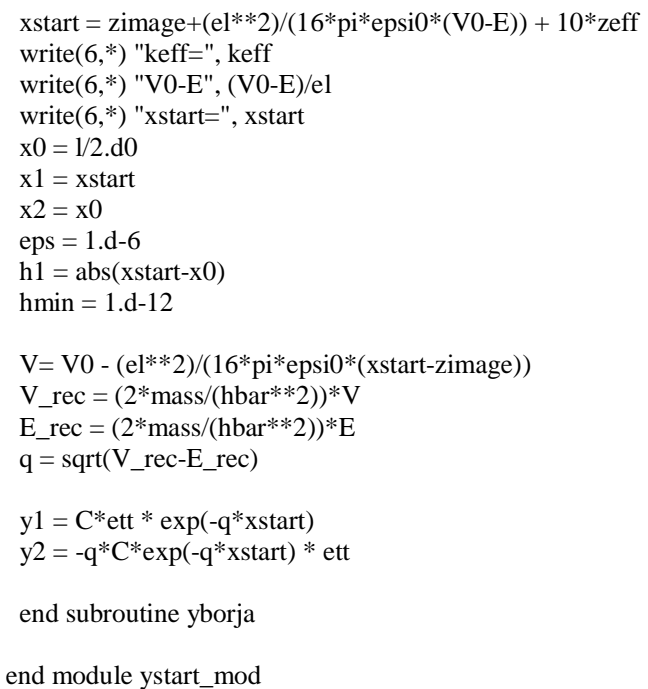

contains

$----11------$

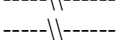

$----11-----$

subroutine odeint(dersub,ystart,x 1,x2,eps,h1,hmin)

implicit none

complex(8), dimension(:), intent(inout) $::$ ystart

real(8), intent(in) :: x1, x2, eps, h1, hmin

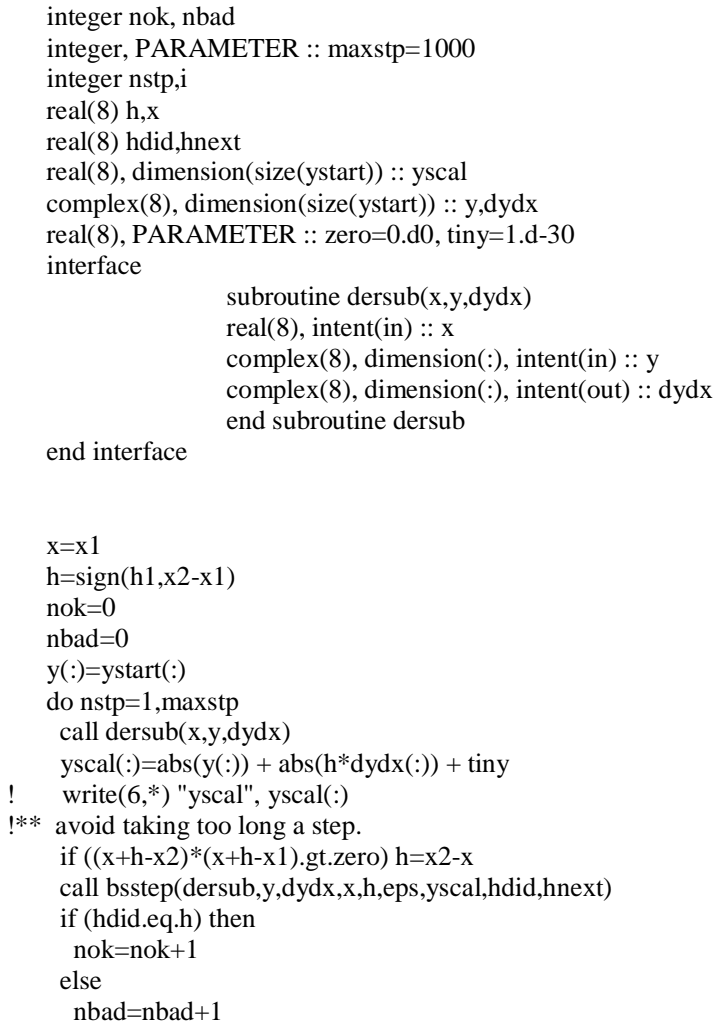

$\mathrm{x}=\mathrm{x} 1$

$\mathrm{h}=\operatorname{sign}(\mathrm{h} 1, \mathrm{x} 2-\mathrm{x} 1)$

nok $=0$

$\mathrm{nbad}=0$

$\mathrm{y}(:)=y \operatorname{start}(:)$

do nstp=1, maxstp

call dersub(x,y,dydx $)$

$\operatorname{yscal}(:)=\operatorname{abs}(y(:))+\operatorname{abs}(h * d y d x(:))+$ tiny

write $(6, *)$ "yscal", yscal(:)

!** avoid taking too long a step

if $((x+h-x 2) *(x+h-x 1)$.gt.zero $) h=x 2-x$

call bsstep(dersub,y,dydx,x,h,eps,yscal,hdid,hnext)

if (hdid.eq.h) then

nok $=$ nok +1

else

nbad $=$ nbad +1 


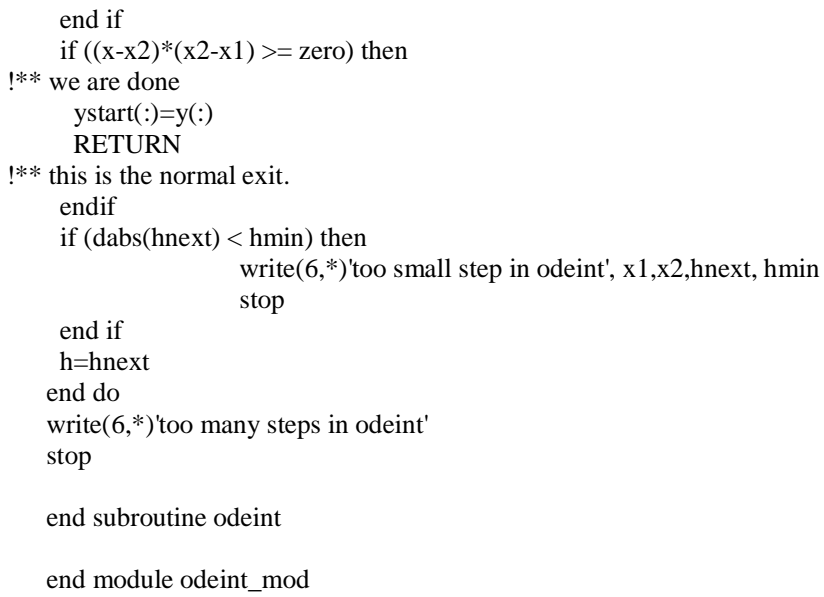

USE param_mod, only: pi, el, epsi0, imun, noll, hbar USE pot_mod, only: V0, mass, zimage contains

subroutine imagederiv $(\mathrm{x}, \mathrm{y}, \mathrm{dydx})$ implicit none

real(8), intent(in) $:: x$$$
\operatorname{real}(8) \mathrm{V}
$$

complex(8), dimension(:), intent(in) :: y complex(8), dimension(:), intent(out) :: dydx

$\mathrm{V}=\mathrm{V} 0-(\mathrm{el} * 2) /(16 * \mathrm{pi} * \mathrm{epsi} 0 *(\mathrm{x}-\mathrm{zimage}))$

$\operatorname{dydx}(1)=y(2)$

$\operatorname{dydx}(2)=((2 * \operatorname{mass} /($ hbar**2) $) *(\mathrm{~V}-\mathrm{y}(3))) * \mathrm{y}(1)$

$\operatorname{dydx}(3)=$ noll $\operatorname{dydx}(4)=-(\operatorname{abs}(\mathrm{y}(1))) * * 2$

end subroutine imagederiv

end module imderiv_mod 


\section{Calculations of the Local Density of States (LDOS)}

To calculate the Local Density of States, we must first calculate the wave vectors and the Green's functions (4-34). Module "green_mod" calculate the wave vectors, energies and finally the Green's functions. These calculated values then can be used in the main program, "el_density" to investigate and derive the Local Density of States, LDOS.

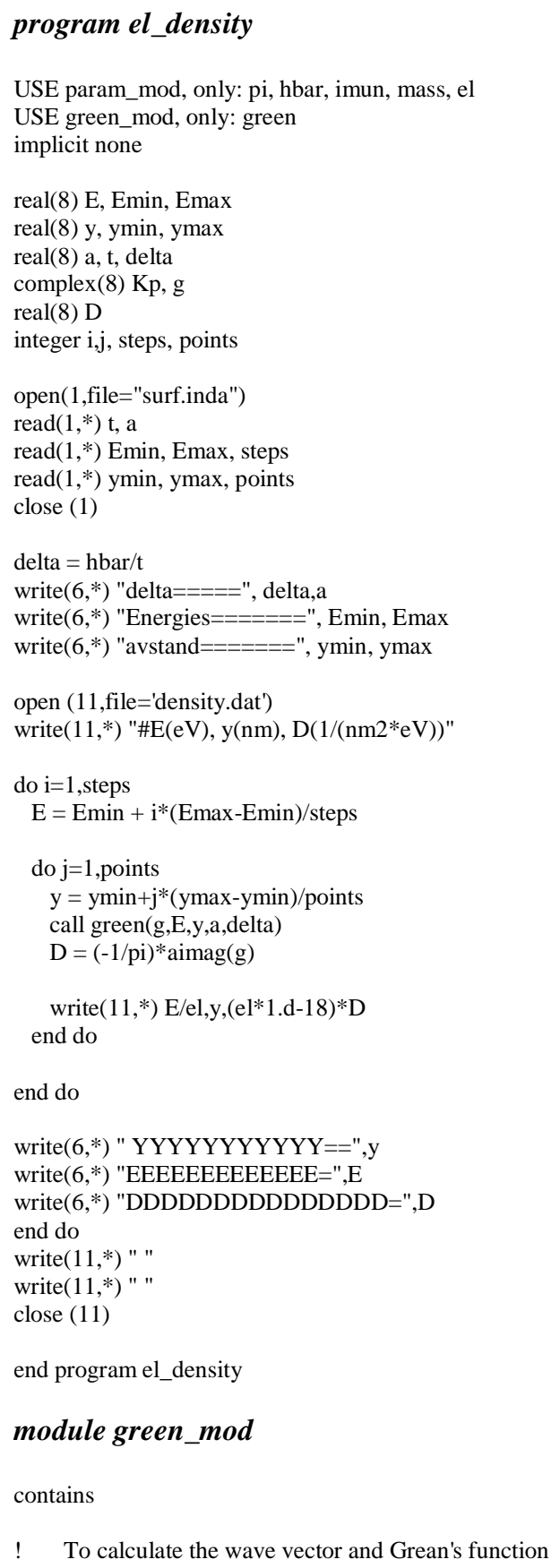


subroutine green (g,E,y,a,delta)

USE param_mod, only: pi, hbar, imun, mass, noll

implicit none

real(8), intent(in) :: E, y, a, delta

complex(8), intent(out) ::g

real(8), PARAMETER :: factor $=10 . \mathrm{d} 3$

integer, PARAMETER $:: n \max =100000$

integer, PARAMETER :: $n \max =1000$

complex(8) Kp, kx, ky

complex(8) term, term1, E1

integer $\mathrm{n}$

$\mathrm{g}=$ noll

do $n=1, n \max$

$\mathrm{E} 1=\mathrm{E}+\mathrm{imun} *$ delta

$\mathrm{kx}=((2 *$ mass $) /(\mathrm{hbar} * * 2)) * \mathrm{E}$

$\mathrm{ky}=(\mathrm{n} * * 2) *\left(\mathrm{pi}^{* * 2}\right) /\left(\mathrm{a}^{* * 2}\right)$

$\mathrm{Kp}=\operatorname{sqrt}(\mathrm{kx}-\mathrm{ky})$

term $=($-imun $*$ mass $) /\left(\mathrm{Kp}^{*}(\mathrm{hbar} * * 2)\right)$

term $1=\operatorname{term} *\left(\sin \left(\mathrm{n}^{*} \mathrm{pi}^{* \mathrm{y} / \mathrm{a})}\right) * * 2\right.$

$\mathrm{g}=\mathrm{g}+$ term 1

write $\left(6,{ }^{*}\right)$ " in i green ar E1",E1

write $(6, *)$ " in i green ar kx", kx

write $(6, *)$ " in i green ar ky", ky

write $\left(6,{ }^{*}\right)$ " in i green ar $\mathrm{kp} ", \mathrm{kp}$

write $(6, *)$ " in i green ar term", term

write $(6, *)$ " in i green ar term1", term1

write $(6, *)$ " in i green ar $\mathrm{g} ", \mathrm{~g}$

write $\left(6,{ }^{*}\right)$ " n\#\#\#\#\#\#\#\#", n

write $(6, *)$ " nmaxpoc000000", nmax

write $(6, *)$ "aimag $(\mathrm{g})=$ ", $\operatorname{aimag}(\mathrm{g})$

write $(6, *)$ "aimag(term 1$) *$ factor $=$ ", $\operatorname{aimag}($ term 1$) *$ factor

if $(\operatorname{abs}(\operatorname{aimag}(g))>=\operatorname{abs}(\operatorname{aimag}($ term $) *$ factor $))$ exit

end do

end subroutine green

end module green_mod

\section{References}


1. Zangwill, Andrew, Physics at surfaces. (Press Syndicate of the University of Cambridge1988), Chap.4.

2. Kittel, Charles, Introduction to Solid State Physics $\left(8^{\text {th }}\right.$ ed.)

3. George B. Arfken \& Hans J. Weber. Mathematical Methods for Physicists. (Academic Press Limited, $4^{\text {th }}$ ed.)

4. Gasiorowicz, Stephan. Quantum Physics. (John Wiley \& Sons, Inc. 1974)

5. William Shockley, Phys. Rev. 56, 317-323 (1939)

6. N. D. Lang and W. Kohn. Phys. Rev. B 1, 4555-4568 (1970)

7. Joel A. Appelbaum and D. R. Hamann, Rev. Mod. Phys. 48, 479-496 (1976)

8. E. V. Chulkov, V. M. Silkin and P. M. Echenique, Surface science 437(1999) 330-352

9. P. M. Echenique, R. Berndt, E. V. Chulkov, Th. Fauster, A. Goldmann and U. Höfer, Surface science reports 52 (2004) 219-317

10. J. M. García, O. Sánchez, P. Segovia, J. E. Ortega, J. Alvarez, A. L. Vázquez de, Parga and R. Miranda, Applied Physics A 61, 609 (1995)

11. O. Sanchez, J. M. Garcia, P. Segovia, J. Alvarez, A. L. Vázquez de Parga, J.E. Ortega, M. Prietsch and R. Miranda, Physical Review B 52, 7894 (1995)

12. A. Beckmann, Ch. Ammer, K. Meinel, H. Neddermeyer, Surface science 432 (1999) L589-L593

13. F. Theilmann, R. Matzdorf, A. Goldmann, Surface science 387 127-135 (1997)

14. J. Li, W. D. Schneider, R. Berndt, O. R. Bryant, S. Crampin, Phys. Rev. Lett. 81, 4464-4467 (1998)

15. R. Matzdorf, A. Goldmann, Surface science 400 (1998) 329-334

16. P. Heimann, J. Hermanson, and H. Miosga, Phys. Rev. B 20, 3059-3066 (1979)

17. F. J. Himpsel and J. E. Ortega, Phys. Rev. B 46, 9719-9723 (1992)

18. S. J. Gurman, Phys. Rev. Lett. 31, 637-639 (1973)

19. J. G. Gay, J. R. Smith, and F. J. Arlinghaus, Phys. Rev. Lett. 42, 332-335 (1979)

20. L. Bürgi, O. Jeandupeux, A. Hirstein, H. Brune, and K. Kern, Phys. Rev. Lett. 81, 5370-5373 (1998)

21. S. M. Driver, D. P. Woodruff, Surface science 560 (2004) 35-44

22. William H. Press, Saul A. Tenkolsky, William T.Vetterling, Brian P. Flannery, Numerical Recipes in Fortran 77. (The Art of Scientific Computing 2nd edition)

23. Y. C. Cheng, \& K. C. Lin, Chinese Journal Physics 26, nr. 4 october1988

24. M. D. Stiles (1997). Generalized Slater-Koster method for fitting band structure

25. http://physweb.spec.warwick.ac.uk/ spsd/, The Warwick STM Gallery

26. http://www.boulder.nist.gov/div853/greenfn/tutorial.html?.html, National Institute of Standard and Technology, NIST

27. Science \& Technology review

28. CEM 924 "Special Topics in Analytical Chemistry"

29. http://www.iap.tuwien.ac.at/www/surface/STM_Gallery/dislocations.html

30. http://en.wikipedia.org/wiki/Crystallographic defect

31. http://www.physik.uni-marburg.de/of/dynamics/wp.html 